\title{
Application of the Zimm-Bragg Model to the Removal of Azo Dyes with Pectin
}

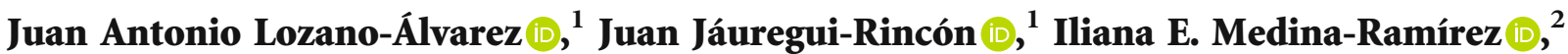 \\ Rogelio Salinas-Gutiérrez $\mathbb{D},{ }^{3}$ Jorge Martín Alférez Chávez $\mathbb{D},{ }^{1}$ \\ and Guadalupe Javier Araiza-Arvilla $\mathbb{D}^{1}$ \\ ${ }^{1}$ Department of Biochemical Engineering, Autonomous University of Aguascalientes, Av. Universidad 940, Ciudad Universitaria, \\ 20100 Aguascalientes, Ags., Mexico \\ ${ }^{2}$ Department of Chemistry, Autonomous University of Aguascalientes, Av. Universidad 940, Ciudad Universitaria, \\ 20100 Aguascalientes, Ags., Mexico \\ ${ }^{3}$ Department of Statistics, Autonomous University of Aguascalientes, Av. Universidad 940, Ciudad Universitaria, \\ 20100 Aguascalientes, Ags., Mexico
}

Correspondence should be addressed to Juan Antonio Lozano-Álvarez; lozanoalvarez@yahoo.com

Received 8 September 2021; Accepted 8 November 2021; Published 23 November 2021

Academic Editor: George Kyzas

Copyright (c) 2021 Juan Antonio Lozano-Álvarez et al. This is an open access article distributed under the Creative Commons Attribution License, which permits unrestricted use, distribution, and reproduction in any medium, provided the original work is properly cited.

\begin{abstract}
In this work, the ability of pectin (Pec) to remove direct red 80 (DR80), Congo red (CR), methyl orange (MO), and methyl red (MR) was studied. The removal percentages under adequate $\mathrm{pH}$ and ionic strength conditions were as follows: DR80 (99.5\%), CR (99.8\%), MO (88.6\%), and MR (68\%), showing that this methodology is efficient to remove azo dyes. The proposed method included the addition of native Pec to the dye aqueous solution and the formation of a gel that occurred when a calcium salt solution was added. This gel retains the molecules adsorbed onto the molecular surface of Pec through hydrogen bonds and electrostatic and hydrophobic interactions. To our knowledge, it is the first time that the Zimm-Bragg model is used to describe the removal of azo dyes with native Pec. This model includes two parameters: $K_{u}$ (nucleation constant), which is related to the tendency exerted by a dye molecule attached to the Pec to bind to other molecules present in the aqueous phase, and $U$ (cooperativity parameter), which determines the aggregation capacity of the dye molecules already attached to the Pec. This model fits the experimental isotherms very well, suggesting that Pec binds single molecules and dye aggregates. The obtained results in the values of $K_{u}$ ranged from $922 \mathrm{~mol} / \mathrm{kg}$ (MR) to $1,157,462 \mathrm{~mol} / \mathrm{kg}$ (CR), and $U$ varied from 2.51 (MR) to 169.19 (MO). These results suggest that the use of Pec is a viable option to remove azo dyes from aqueous effluents and that the Zimm-Bragg model fits adequately the isotherms of dyes that have a high tendency to form aggregates.
\end{abstract}

\section{Introduction}

The azo dyes correspond to a group of synthetic compounds, which are characterized by including the azo group $(-\mathrm{N}=\mathrm{N})$ in their structure and by imparting a wide variety of colors in the different substrates where they are applied. These compounds are widely used in the cosmetic industry, photography, and optoelectronics, in the staining of different biological tissues and cells, in the dyeing of leather, in the textile industry, etc. [1]. However, it is known that these dyes are carcinogenic and it has been reported that when they are degraded anaerobically, toxic amines are produced [2]. Due to these adverse effects, alternative methods have been sought in the staining process. One of these methods is the use of supercritical fluids for the fixation of different dyes to several fibers [3]. However, only supercritical carbon dioxide has been applied to reactive and disperse azo dyes, and this has limited application [4-6], and unfortunately, nowadays the wet staining process is still used in the dyeing of different textile fibers [7]. As a consequence, this generates 
environmental problems by discharging effluents with these dyes to different water bodies. Moreover, the problem becomes more complex because it is known that from of all of the dyes produced annually, azo dyes correspond to 65$70 \%$ approximately [8].

Given these facts, sustainable methods are required to decrease the concentration of these dyes in wastewater. Among these methodologies, the use of biopolymers such as pectin $(\mathrm{Pec})$ is considered a good strategy due to its high availability in nature [9].

This heteropolysaccharide is formed by a polygalacturonic acid backbone that can form esters with methanol (high methoxyl pectins (HMP)) or form sodium, potassium, calcium, and ammonium salts mainly (low methoxyl pectins (LMP)). Additionally, it contains sugar residues such as rhamnose, galactose, arabinose, fucose, mannose, xylose, and glucose among others and is used as a thickener and gelling agent in the food industry, due to its high capacity to form gels in the presence of high concentrations of sucrose (HMP) and the presence of calcium salts (LMP) [10].

Recently, different compounds obtained from Pec including hydrogels, grafting products, and nanocomposites have been used to remove several pollutants such as metal ions $\left(\mathrm{Pb}^{+2}, \mathrm{Cd}^{+2}, \mathrm{Cu}^{+2}, \mathrm{Zn}^{+2}, \mathrm{Cs}^{+1}\right.$, and $\left.\mathrm{Ce}^{+3}\right)$ [11-16] and dyes such as methylene blue [17-22], crystal violet, methyl orange, Eriochrome black T, and basic yellow 28 [23-25] and other pollutants such as cetyl pyridinium and benzethonium dissolved as chloride salts in aqueous medium [26]. However, the synthesis of different Pec derivatives implicates high production costs and decreases the biodegradability of these compounds. For this reason, this work is focused on the use of Pec from citrus peel without any chemical modification to remove various azo dyes, avoiding these drawbacks.

In a more specific way, it has been reported that polygalacturonic acid regions of LMP react with $\mathrm{Ca}^{+2}$ ions in a similar manner to polyguluronic acid contained in alginic acid, with the consequent formation of calcium pectinate (Pec-Ca) [27]. In this sense, this process was carried out in two steps: (1) the interaction of the dyes with Pec and (2) the subsequent gel formation with $\mathrm{Ca}^{+2}$ ion, to remove the following azo dyes: direct red 80 (DR80), Congo red (CR), methyl orange (MO), and methyl red (MR) from an aqueous environment. On the other hand, it is remarkable that in most of these reports, the Langmuir and Freundlich models are among the most widely used [28, 29], but no article has the application of the Zimm-Bragg model which has been considered for Pec-dye systems [30]. Therefore, the main goal of this work was the application of this model to the experimental isotherms obtained when using Pec in the removal of azo dyes from the aqueous phase.

\section{Materials and Methods}

2.1. Materials. Pectin from citrus peel (galacturonic acid $\geq$ $74.0 \%), \mathrm{CaCl}_{2}$ (97\%), and the dyes DR80, CR, MO, and MR were purchased from Sigma-Aldrich (St. Louis, MO, USA) and were used without further purification. Distilled water, $\mathrm{NaOH}, \mathrm{HCl}$, and $\mathrm{NaCl}$ were provided by JT Baker (México State, México).
2.2. Optimization of Dye Removal Conditions. A Pec hydrated solution $(1 \% w / v)$ was prepared at $\mathrm{pH}=12$, and it was incubated at room temperature $\left(27 \hat{\mathrm{A}}^{\circ} \mathrm{C}\right)$ to favor the hydrolysis of the methoxyl ester groups attached to this polysaccharide. After, an aliquot of dye solution was added to the polymer. The final Pec concentration was set to $0.5 \% \mathrm{w} / v$. In addition, the $\mathrm{pH}$ and ionic strength (IS) values were adjusted to adequate values with $\mathrm{NaOH}, \mathrm{HCl}$, and $\mathrm{NaCl}$ aqueous solutions, and a volume of $100 \mathrm{~mL}$ of $\mathrm{Pec}-$ dye solution was obtained (with a final dye concentration of $100 \mathrm{mg} / \mathrm{L}$ ). Due to differences in dye solubility, the $\mathrm{pH}$ values were assigned as follows: $\operatorname{DR} 80(4,6.5$, and 9), CR and $\operatorname{MO}(4,8$, and 12), and $\operatorname{MR}(7,9.5$, and 12). The final values of IS were $0.04 \mathrm{M}, 0.07 \mathrm{M}$, and $0.1 \mathrm{M}$ for all of the dyes. After that, $30 \mathrm{~mL}$ of $\mathrm{CaCl}_{2}(5 \% w / v)$ was added producing a gel, and a volume of $200 \mathrm{~mL}$ was completed with distilled water and incubated at $27^{\circ} \mathrm{C}(24 \mathrm{~h})$ in the darkness. An aliquot of the supernatant was centrifuged at $9000 \mathrm{~g}$ (20 $\mathrm{min})$, and the dye was determined by UV-visible spectroscopy in a Thermo Scientific spectrophotometer model evolution 201 that measured the absorbance from each dye at its maximum absorption wavelength, which was located between 400 and $800 \mathrm{~nm}$, and all experiments were performed in triplicate. The percentage of removal was obtained with the following equation:

$$
\operatorname{Removal}(\%)=\frac{\left(C_{\mathrm{i}}-C_{\mathrm{eq}}\right) \times 100}{C_{\mathrm{i}}},
$$

where $C_{\mathrm{i}}$ and $C_{\mathrm{eq}}$ are the initial and final dye concentration values $(\mathrm{mg} / \mathrm{L})$, respectively.

2.3. Removal Isotherms. Several dye solutions with different concentration values within the range of $0-4000 \mathrm{mg} / \mathrm{L}$ were prepared to take into account the specific $\mathrm{pH}$ and IS values of each dye determined from the previous section. A Pec solution aliquot with the same values of $\mathrm{pH}$ and IS was added to an equivalent volume of each dye solution, and the final concentration of each dye ranged from 0 to $2000 \mathrm{mg} / \mathrm{L}$. The resulting solution was mixed vigorously for $15 \mathrm{~min}$, and the addition of an aqueous solution of $\mathrm{CaCl}_{2}$ at $5 \%(w / v)$ produced the compound Pec-dye-Ca, whose rheological behavior changed from a typical viscous liquid to a rigid gel. The volume was completed to $200 \mathrm{~mL}$ with distilled water, and the gel immersed in the aqueous phase was incubated at $27^{\circ} \mathrm{C}$ for $24 \mathrm{~h}$. Finally, the dye concentration $\left(C_{\mathrm{eq}}\right)$ in the supernatant was quantified as described in the previous section. All removal isotherms were carried out in triplicate.

2.4. Application of the Adsorption Models to Experimental Isotherms. The Langmuir model is described by the equation:

$$
q_{e}=q_{m} \cdot K_{\mathrm{L}} \cdot \frac{C_{\mathrm{eq}}}{1+K_{\mathrm{L}} \cdot C_{\mathrm{eq}}},
$$

where 
(i) $q_{e}$ refers to the mass of dye retained per unit mass of $\mathrm{Pec}$ (mol of dye/mol of Pec), which is obtained experimentally by the following equation:

$$
q_{e}=\frac{\left(C_{\mathrm{i}}-C_{\mathrm{eq}}\right) \times V}{m} .
$$

Both $C_{\mathrm{i}}$ and $C_{\mathrm{eq}}$ represent the dye concentration in the liquid phase at the start and the equilibrium state (mol of dye/L), respectively, $V$ represents the volume of dye solution (L), and $m$ is the mass of Pec (mol) needed to remove each dye.

(ii) $K_{\mathrm{L}}$ is the Langmuir constant, with the following units $(\mathrm{L} / \mathrm{mol}$ of dye)

(iii) $q_{m}$ is the maximum adsorption capacity, which is defined as the sufficient amount of dye to form a monolayer on the surface of the Pec (mol of dye/ mol of Pec)

By rearranging the Langmuir equation, the following linear form is obtained:

$$
\frac{C_{\mathrm{eq}}}{q_{e}}=\frac{1}{K_{\mathrm{L}} \cdot q_{m}}+\frac{1}{q_{m}} C_{\mathrm{eq}} .
$$

In this sense, when $C_{\mathrm{eq}} / q_{e}$ is plotted as a function of $C_{\mathrm{eq}}$, a straight line with an intercept $1 /\left(K_{\mathrm{L}} \cdot q_{m}\right)$ and slope $1 / q_{m}$ is found.

The theoretical parameters of the Langmuir model can be obtained by fitting the linear form (4) to a set of experimental data. The linear regression process allows estimating the intercept and the slope of a linear model and provides statistical information about the estimated parameters as well as the linear model, such as the standard deviation of the estimated parameters and the model. In this work, the statistical software R [31] was used for applying the linear regression to the experimental data and for estimating the $K_{\mathrm{L}}$ and $q_{m}$ parameters.

The equation of the Freundlich model is as follows:

$$
q_{e}=K_{\mathrm{F}} \cdot C_{\mathrm{eq}}^{1 / n}
$$

where

(i) $q_{e}$ and $C_{\text {eq }}$ were defined previously

(ii) $K_{\mathrm{F}}$ is Freundlich's constant (mol of dye $/ \mathrm{mol}$ of $\mathrm{Pec})\left(\mathrm{mol}\right.$ of dye/L) ${ }^{-1 / n}$

(iii) $1 / n$ is the adsorption intensity number

The application of the logarithm function to equation (5) allows obtaining a linear form given by the following expression:

$$
\log \left(q_{e}\right)=\log \left(K_{\mathrm{F}}\right)+\left(\frac{1}{n}\right) \log \left(C_{\mathrm{eq}}\right)
$$

In the same way as the linear form of the Langmuir model, a graph of $\log \left(q_{e}\right)$ versus $\log \left(C_{\text {eq }}\right)$ refers to a straight line with an intercept defined by $\log \left(K_{\mathrm{F}}\right)$ and a slope $1 / n$. The linear regression method can be applied to a set of experimental data to estimate the parameters of the linear form of the Freundlich model (6), as was done in this work.

The Zimm-Bragg model has been applied and reported as an adsorption model by our group [32]. In this work, the theoretical parameters of the Zimm-Bragg model, $K_{u}$ and $U$ (nucleation and cooperativity parameters, respectively), were obtained by using the nonlinear regression method in the experimental isotherms. The description of this model as well as its application to the experimental data is shown in Section 3.2.

Additionally to the estimated parameters, several goodness-of-fit measures are reported such as the coefficient of determination $\left(R^{2}\right)$, the average relative error, and the standard deviation for the three models applied to the experimental isotherms.

2.5. Visible Studies. The electronic absorption spectra of all of the dyes were obtained in a Thermo Scientific spectrophotometer (evolution 201) in the range of 400 to $800 \mathrm{~nm}$, and the effect of the following variables on them was studied.

2.5.1. Effect of the $p H$. Several aqueous solutions of each dye $\left(1 * 10^{-5} \mathrm{M}\right)$ were prepared in the range of $\mathrm{pH}$ values between 4 and 12 and after the visible spectrum of each dye was obtained.

2.5.2. Effect of Dye Concentration and the Presence of Pec. Different dye solutions at different concentration values (5-200 mg/L) were prepared under the optimal removal conditions of $\mathrm{pH}$ and ionic strength corresponding to each dye. This was done in the presence and absence of Pec $(1 \% w / v)$, to observe the effect of this heteropolysaccharide on the electronic spectrum of each dye.

2.5.3. Effect of the Formation of the Pec-Ca Gel. Some of the Pec-dye solutions prepared in the previous section were used to obtain the Pec-dye-Ca product. This compound was prepared with the addition of $\mathrm{CaCl}_{2}(5 \% \mathrm{w} / \mathrm{v})$ to the Pec-dye aqueous solution and after an incubation period of $24 \mathrm{~h}$ at $27 \hat{\mathrm{A}}^{\circ} \mathrm{C}$. The resultant gel was analyzed through visible spectrophotometry.

In the last experiments (Sections 2.5.2 and 2.5.3), the path length of the cell was modified to adjust the absorbance at lower values than the maximum detection limit of the spectrophotometer; consequently, the absorbance values observed do not correspond in a linear relationship with the concentration of each dye.

2.6. Infrared and Raman Studies. FT-IR spectra of all dyes, $\mathrm{Pec}-\mathrm{Ca}$, and Pec-dye-Ca products $\left(4000-600 \mathrm{~cm}^{-1}\right)$ were obtained in a Jasco model 4600 series equipment (in an attenuated total reflectance mode), with a resolution set at 
$1 \mathrm{~cm}^{-1}$, and each spectrum resulted from 64 scans. The Raman spectra $\left(2000-400 \mathrm{~cm}^{-1}\right)$ of the same dyes and compounds were obtained in a Micro-Raman system (Horiba, model LabRAM HR Evolution) equipped with a $785 \mathrm{~nm}$ laser. The power used was $5 \%$, and the integration time was 15 seconds. The different Pec-dye-Ca products were obtained as described in Section 2.3, and the only one sample considered to be analyzed was the last of each removal isotherm, corresponding to the maximum dye concentration value considered $(2000 \mathrm{mg} / \mathrm{L})$. Once the gel of each dye was obtained, it was washed with distilled water (5 times) to remove the unbound dye molecules. Then, the gel was frozen, lyophilized, and ground to obtain a fine powder. Finally, the FT-IR and Raman spectra were obtained in the same manner described for the dyes.

\section{Results and Discussion}

3.1. Optimization of Dye Removal Conditions. The dye removal efficiency of DR80, CR, MO, and MR azo dyes with $\mathrm{Pec}$ was evaluated at different $\mathrm{pH}$ and IS values (Figure 1).

From Figure 1(a), it is observed that as $\mathrm{pH}$ is increased at high IS $=0.1 \mathrm{M}$, the removal of DR80 is notably augmented, and inversely, at low IS values $(0.04 \mathrm{M})$, it tends to decrease slightly. As can be deduced from Figure 2, the DR80 molecule has a negative charge at high $\mathrm{pH}$ values, and the increase in this parameter favors its solubility. However, also, the repulsion between the dye molecules and the residues of galacturonic acid deprotonated attached to Pec is increased.

Nevertheless, it has been reported that these galacturonate groups included in the smooth region of the Pec structure are involved in the formation of calcium pectate (Pec-Ca), which is the final product that retains DR80 and diminishes its concentration in the aqueous medium. In this sense, it has been reported that polygalacturonate regions similarly react with $\mathrm{Ca}^{+2}$ ions as the guluronate groups do with the alginic acid, promoting the formation of a very stable Pec-Ca gel [27]. Moreover, they proposed that $\mathrm{Ca}^{+2}$ ions promote the formation of dimers from this polysaccharide which will subsequently polymerize to form a network of this compound, which in the solid state has shown a similar conformation to that reported for alginate. These findings suggest an analogous manner of gelling observed for both Pec and alginic acid [33]. It has been found that different azo dyes are adsorbed onto the alginic acid molecular surface, and after $\mathrm{Ca}^{+2}$ addition, these azo dyes are trapped into the network of calcium alginate [34]. In the same order, we propose a similar mechanism between DR80 and Pec that can be explained based on the same structural basis from the alginate molecule.

Additionally, it has been reported that Pec consists of two principal regions: the "smooth region" and the "hairy region" [10]. The former contains a large number of galacturonate residues with ionizable carboxyl groups at high $\mathrm{pH}$ values. The hairy region contains mostly neutral sugars such as rhamnose, arabinose, xylose, galactose, fucose, mannose, and glucose among others, which results in a low charge density of this polysaccharide, and consequently, the repulsion exerted by Pec on DR80 is less than the result of the alginic acid-DR80 system. Even more, when the Pec$\mathrm{Ca}$ gel is formed, the insertion of the $\mathrm{Ca}^{+2}$ ions between the Pec chains promotes a decrease in the effective charge of Pec-Ca and favors the binding of DR80 to Pec.

In the same order, DR80 molecules interact with Pec through hydrogen bonds between the hydroxyl groups, oxygen atoms from the hemiacetal, and glycosidic bonds attached to Pec molecules and the sulfonate, hydroxyl, azo, and amido groups from this dye.

It has been found that high IS values from an aqueous environment favor the formation of aggregates in dyes whose molecules have aromatic rings that originate a planar geometry [35]. Additionally, Oakes and Dixon have proposed that the higher the number of aromatic rings in dye molecules, the bigger the dye aggregates, due to the $\pi-\pi$ stacking interactions [36]. From Figure 2(a), it can be observed that the DR80 molecule has two naphthyl and four phenyl rings, which suggests that this dye can form aggregates. It has been encountered that the existence of hydrophobic interactions between the $\pi$ electrons from dye aromatic rings and hydroxyl groups attached to polysaccharides such as cellulose is involved in the binding of dye molecules to this polymer [37].

In this sense, the hairy region included in the Pec molecule is similar to some regions found in cellulose, due to the presence of neutral sugars that structurally resemble the $\beta$ $D$-glucose contained in that polysaccharide, which suggests that this type of interactions contributes considerably to the binding of DR80 to the Pec. In addition, the smooth region included in the Pec structure can also interact with this dye, but in a more limited manner due to the low accessibility of DR80 molecules to this part of the Pec structure. All these contributions which promote the binding of DR80 to Pec resulted in a high removal efficiency (99.5\%) at $\mathrm{pH}=9$ and $\mathrm{IS}=0.1 \mathrm{M}$.

In Figure 1(b), the variation of the removal efficiency of $\mathrm{CR}$ is shown as a function of $\mathrm{pH}$ and IS for the system Pec$\mathrm{CR}-\mathrm{Ca}$. It is observed that in the extreme $\mathrm{pH}$ values, the removal of this dye shows the maximum values. At $\mathrm{pH}=4$, the $\mathrm{CR}$ tends to precipitate due to the proximity of this $\mathrm{pH}$ value with its $\mathrm{pKa}$ (4.1) [38]. Despite this, CR was removed by Pec, which demonstrates that this biopolymer can remove both soluble and insoluble CR molecules, and as a consequence, $\mathrm{Pec}$ has been used as a coagulating and flocculating agent $[39,40]$.

On the other hand, an increase in the $\mathrm{pH}$ causes the deprotonation of the $\mathrm{CR}$. Consequently, its solubility is augmented, in such a manner that at $\mathrm{pH}=8$, the removal efficiency is decreased as a result of the repulsion between the galacturonate residues from $\mathrm{Pec}$ and the molecules of CR with a negative charge ( $92 \%$ of dye removal at $\mathrm{pH}=8$ ). Interestingly, an alkaline medium does not decrease the removal efficiency of this dye but rather increases it. It has been reported that $\mathrm{CR}$ molecules form aggregates at high $\mathrm{pH}$ values, and there is even evidence of supramolecular structures of this dye [41]. In this sense, the bonding of these "supramolecular units" onto the Pec molecular surface will result in a high removal percentage. These findings suggest that despite the repulsion between Pec and CR, there are 


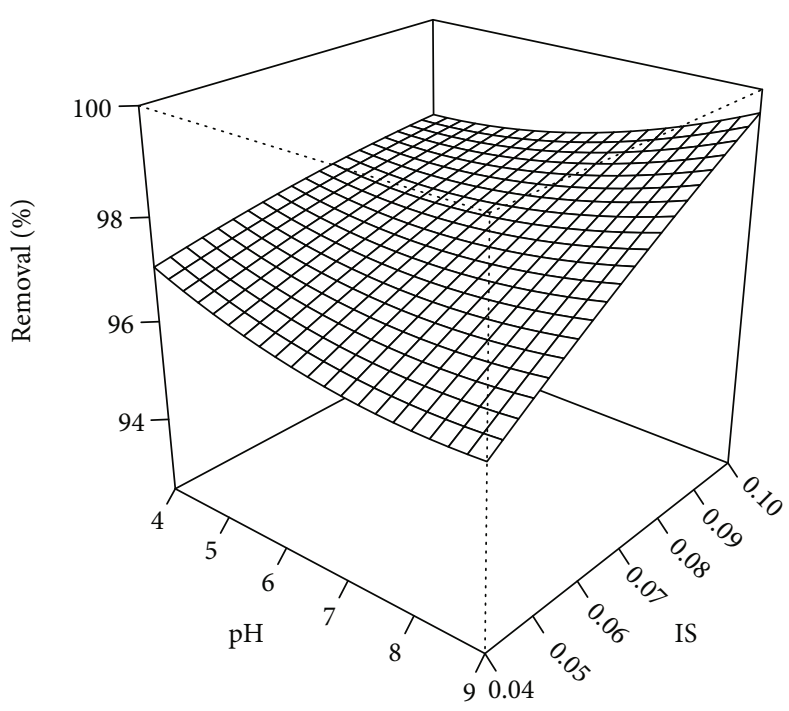

(a)

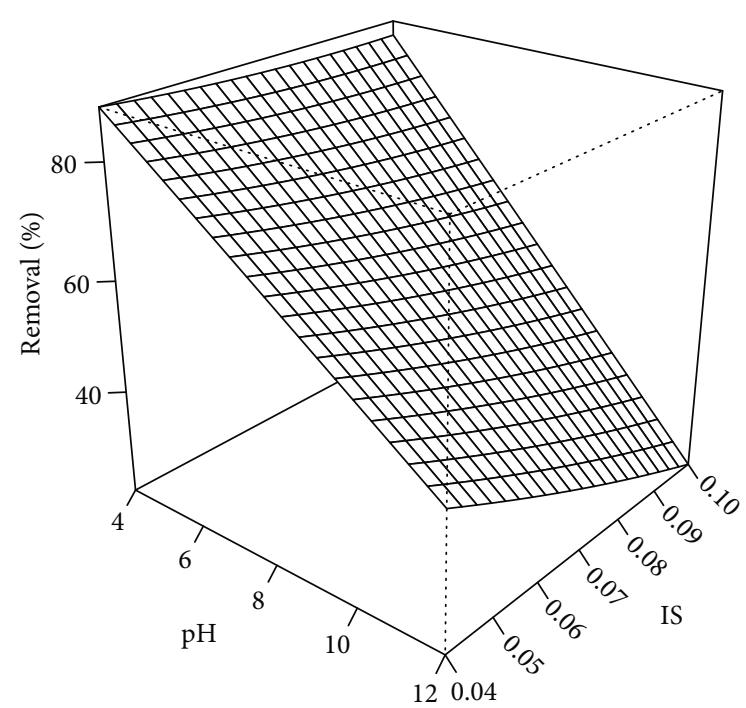

(c)

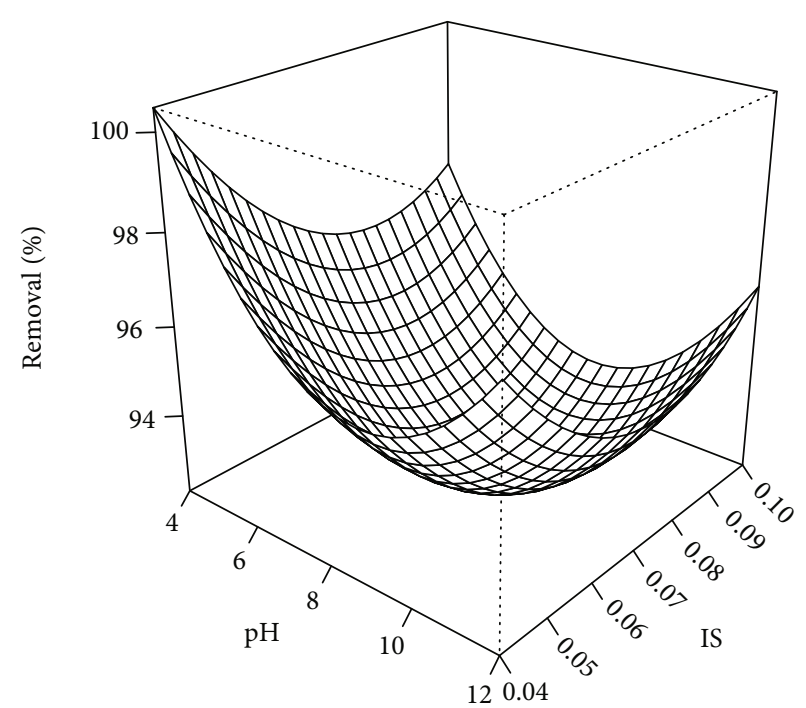

(b)

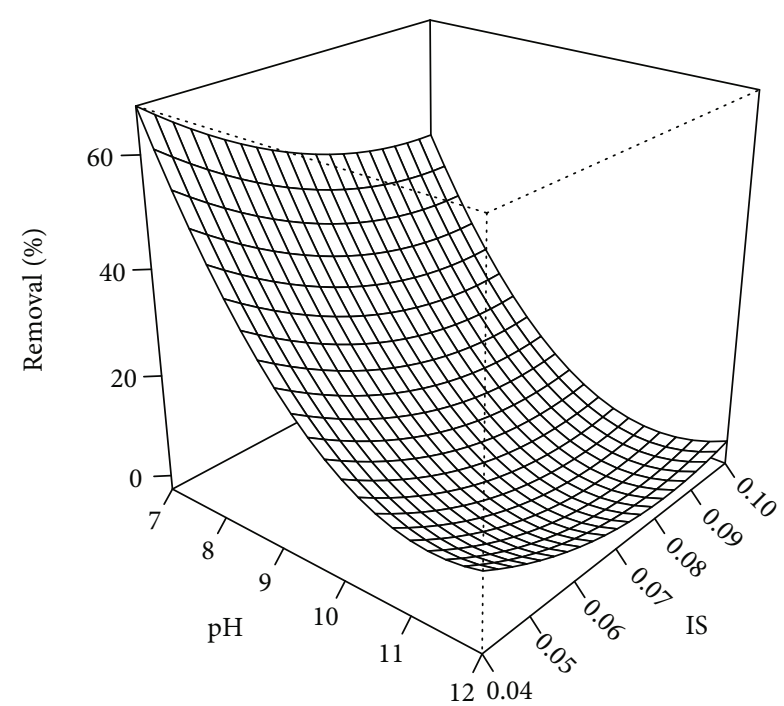

(d)

Figure 1: Effect of $\mathrm{pH}$ and IS on the removal efficiency for the systems: (a) Pec-DR80-Ca, (b) Pec-CR-Ca, (c) Pec-MO-Ca, and (d) PecMR-Ca.

hydrogen bonds between the functional groups of CR (amino, sulfonate, and azo) and the groups attached to the pectin monosaccharides (hydroxyl, carboxylate, oxygen atoms involved in hemiacetals, and glycosidic bonds). As previously mentioned, the hairy region can bind to CR molecules through hydrophobic interactions, which increases the removal efficiency of this dye by Pec.

Otherwise, it can be seen in Figure 1(b) that as the IS decreases, the removal efficiency of the CR increases slightly. To explain this fact, it should be mentioned that for maximum dye removal, Pec must remain soluble in the aqueous medium. In this sense, Wang et al. [42] reported that an increase in the content of electrolytes at basic $\mathrm{pH}$ reduced the solubility of this polysaccharide. Therefore, the highest removal value obtained was at IS of $0.04 \mathrm{M}$. All these findings resulted in a removal efficiency of $99.8 \%$ at $\mathrm{pH}=4$ and $\mathrm{IS}=0.04 \mathrm{M}$.
Figure 1(c) shows the effect of $\mathrm{pH}$ and IS on the removal efficiency of $\mathrm{MO}$ by Pec. As can be seen, as the $\mathrm{pH}$ increases, the removal efficiency decreases notably. In this sense, by inspecting Figures $2(a)-2(c)$, it can be noted that the MO shows several differences in its structure and size concerning the DR80 and CR molecules. For example, the number of functional groups contained in the MO is less than those attached to the other two dyes (MO has only one sulfonate, dimethylamino, and azo groups). This means that at $\mathrm{pH}=4$, $\mathrm{MO}$ molecules are partially deprotonated and with a little negative charge, due to the $\mathrm{pKa}$ value corresponding to $\mathrm{MO}$ which is 3.40 [38]. But this negative charge will increase as the $\mathrm{pH}$ changes to values higher than 4 , until $\mathrm{pH}=12$, where the effective net charge of each MO molecule will be -1 , which will decrease their affinity for Pec due to the repulsion existing between $\mathrm{MO}$ and Pec. This occurs even though the formation of Pec-MO-Ca decreases the negative charge 


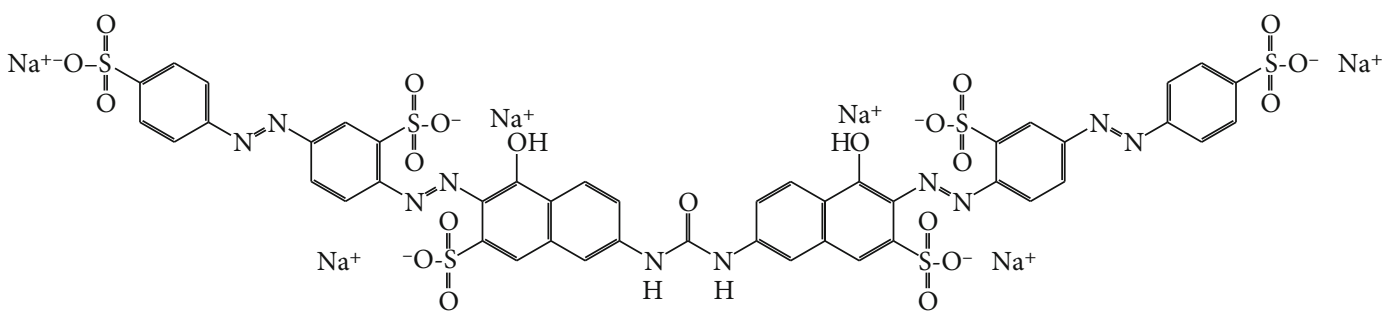

(a)<smiles></smiles>

(b)<smiles>CN(C)C1CCC(NNC2CCC([Si]([NH2+])(=O)[O-])CC2)CC1</smiles>

(c)<smiles>CN(C)c1ccc(N=Nc2ccccc2C(=O)O)cc1</smiles>

(d)

FIgURe 2: The structure of different azo dyes used in this study: (a) DR80, (b) CR, (c) MO, and (d) MR.

of Pec, suggesting that the interactions between $\mathrm{MO}$ and $\mathrm{Pec}$ are weaker than those existing in the Pec-DR80-Ca and Pec-CR-Ca systems.

Moreover, although sulfonate groups of different types of dyes are known to allow them to bind to fibers such as cotton, which contain hydroxyl groups onto their molecular surface [2], it was found that the affinity of $\mathrm{MO}$ for $\mathrm{Pec}$ is lower than that observed in RD80 and CR dyes, despite the similarity of the hairy regions included in Pec with the cellulose structure. In addition, the number of hydrogen bonds between the sulfonate, dimethylamino, and azo groups belonging to $\mathrm{MO}$ and the functional groups attached to the Pec molecule is less than those involved in the Pec-DR80$\mathrm{Ca}$ and Pec-CR-Ca compounds.

On the other hand, as was mentioned before, the aggregation capacity of the dyes decreases as the molecular weight, the number, and the size of the aromatic rings included in the molecular structure are decreased [36]. In this sense, the MO molecule only contains 2 phenyl rings, and as a consequence, the size of aggregates adsorbed to hydrophobic regions of $\mathrm{Pec}$ is small; this results in low removal efficiency. In the same way, it has been reported that an increase in IS promotes hydrophobic interactions between dyes and polysaccharides [35]. As can be seen in Figure 1(c), the effect of the IS on the removal efficiency is almost slight, due to the small size and molecular weight, which as mentioned previously results in lower removal percentages of MO by Pec. Considering all of these findings, a maximum removal value of $\mathrm{MO}$ corresponding to $88.6 \%$ was obtained at $\mathrm{pH}=4$ and $\mathrm{IS}=0.04 \mathrm{M}$.

Figure 1(d) depicts the influence of $\mathrm{pH}$ and IS on the removal efficiency of the Pec-MR-Ca system. As can be observed, the $\mathrm{pH}$ affects in the same manner both $\mathrm{MR}$ and $\mathrm{MO}$, except that this effect is more pronounced in MR dye, which results in lower removal values of this compound than those data found in the Pec-MO-Ca system. Figure 2 shows a high similarity between the structures of $\mathrm{MO}$ and $\mathrm{MR}$, and it is evident that their structures are less complex than those corresponding to DR80 and CR.

In the same way, the chemical structures of $\mathrm{MR}$ and $\mathrm{MO}$ molecules are similar, except that in the case of MR, the sulfonate group in para position with respect to the azo group was substituted by a carboxyl group in the ortho position. This change will give rise to a $\mathrm{pKa}=4.8$ [38], which suggests that at $\mathrm{pH}=7$, the dye molecules are partially deprotonated and have a considerable negative charge that repels the Pec, in such a way that at high $\mathrm{pH}$ value, the repulsion force between MR and Pec will be bigger and this will lead to a decrease in the removal of this dye.

Interestingly, it is notable that the exchange of the sulfonate group in MO by carboxylate anion in MR affects the affinity of MR by Pec. This change causes a decrease in the ability of MR to interact with Pec through hydrogen bonding and a decrease in the number of aggregates of this dye, which diminishes the removal of MR by Pec. Additionally, the molecular weight of MR $(291.28 \mathrm{~g} / \mathrm{mol})$ is smaller than 
that corresponding to $\mathrm{MO}(327.33 \mathrm{~g} / \mathrm{mol})$, which suggests that although the number of binding sites in Pec is the same for both compounds, the removal of the MR would be less in terms of effective mass.

On the other hand, Figure 1(d) shows that as the IS increases, the removal efficiency diminishes, and this trend is more pronounced at $\mathrm{pH}=7.0$. Unlike the other dyes, it is observed that the addition of $\mathrm{Na}^{+}$and $\mathrm{Cl}^{-}$ions to the Pec-MR aqueous system promotes competition between the dye molecules and these ions for Pec, which decreases the interaction of MR with this biopolymer. This effect is less noticeable at high $\mathrm{pH}$ values due to a high number of hydroxyl anions in the aqueous environment and the greater repulsion between the negative MR anion and the carboxylate groups from Pec. All these factors resulted in a removal efficiency of $68 \%$ at $\mathrm{pH}=7$ and IS $=0.04 \mathrm{M}$.

\subsection{Removal Isotherms and Application of the Models to} Experimental Isotherms. As mentioned before, the Langmuir and Freundlich adsorption models have been applied widely in published reports to describe the experimental dye removal isotherms obtained when using pectin derivatives, nanocomposites, and blends of this polysaccharide with other compounds.

In this sense, these two models were used to describe the experimental isotherms of the four systems studied in this work. The linear form of the Langmuir model was applied to experimental data of all systems, and the parameter values and plots that resulted from this procedure are shown in Table 1 and Figures 3(a)-3(d), respectively. Figure 3(a) shows that this model did not fit the experimental data, because a graph with a decreasing behavior and an asymptotic tendency towards a minimum resulted when a $C_{\mathrm{eq}} / q_{e}$ versus $C_{\mathrm{eq}}$ plot was drawn. If this model fitted to the experimental isotherm, a straight line with a positive slope would be observed with positive values of the parameters $K_{\mathrm{L}}$ and $q_{m}$.

Contrary to the above, Table 1 shows negative values of $K_{\mathrm{L}}$ and $q_{m}$. These results were obtained because the Langmuir model only considers the formation of a monolayer, and it can be successfully applied to type I (b) isotherms of the IUPAC classification [43] or type " $\mathrm{L}$ " isotherm [44]. This type of isotherm is characterized by a gradual increase in the value of $q_{e}$ as the concentration of dye in the solution $\left(C_{e q}\right)$ increases and consequently reaches the saturation of the adsorbate binding sites in the Pec. This saturation phenomenon is shown as a plateau in the experimental adsorption isotherm and does not consider the interaction between adsorbate molecules and the formation of dye aggregates, nor their binding to the molecular surface of the Pec. In contrast, the Zimm-Bragg model considers that the dye molecules undergo aggregation and form multimeric molecular entities that bind to the Pec. This coincides with that reported by Hinz [45], which indicates that the sigmoid isotherm results from the phenomenon called "cooperative adsorption," which implies that once the dye molecules formed a monolayer on the adsorbent $(\mathrm{Pec})$, they promote the binding of other molecules found in the aqueous solution, which can be adequately described by the latest model. The above described occurs in the first three systems (Pec-DR80-Ca, Pec-CR-Ca, and Pec-MO-Ca) but with a different degree of magnitude of the values obtained from the $K_{\mathrm{L}}$ and $q_{m}$ parameters of these systems (see Table 1 and Figures 3(a)-3(c)), where the discrepancy between the theoretical lines and the experimental data is evident.

On the other hand, the corresponding values of $K_{\mathrm{L}}=$ $888.02 \mathrm{~L} / \mathrm{mol}, q_{m}=875.29 \mathrm{~mol}$ of dye $/ \mathrm{mol}$ of Pec, and $R^{2}=$ 0.776 from the Pec-MR-Ca system could suggest that the Langmuir model fits moderately with the experimental data. In this sense, Figure 3(d) shows that only a fraction of the experimental data is described by the theoretical line, and there are several points at a low concentration range that are not close to this line. These findings indicate that this model does not fit adequately to the experimental isotherm of the Pec-MR-Ca system. Additionally, Figure 3(d) shows a slight sigmoid behavior, which indicates a small but considerable aggregation capacity of the MR in this system, as it will be proposed later.

Table 2 includes the estimated values of the Freundlich parameters $\left(K_{\mathrm{F}}\right.$ and $\left.n\right)$, and Figures $4(\mathrm{a})-4(\mathrm{~d})$ show the theoretical $\log \left(q_{e}\right)$ versus $\log \left(C_{e q}\right)$ graphs obtained with the parameters included in this table and with the experimental data, to determine whether this model fits with the experimental isotherms of the four systems studied.

Figure 4(a) shows the $\log \left(q_{e}\right)$ versus $\log \left(C_{\text {eq }}\right)$ graph from the Pec-DR80 system. This figure and the value of the coefficient of determination $R^{2}=0.983$ reported in Table 2 indicate an "apparent" good fit of this model to experimental data. However, it should be noted that these data do not show a linear behavior, but rather a sigmoid trend is observed. From this same table, the value of $K_{\mathrm{F}}=1,127,755,625$ (mol of dye/mol of $\mathrm{Pec})(\mathrm{mol} \text { of dye } / \mathrm{L})^{-1 / n}$ is extremely high, which is impossible to achieve on a practical level. In addition, the value of $1 / n=$ 1.274 suggests that the adsorption is unfavorable [46]. This information contrasts with the results of the previous section (see Section 3.1), where it was found that DR80 was removed efficiently with Pec.

In Figure 4(b), it can be seen that the Pec-CR-Ca system has similar behavior to that of Figure 4(a), but with a marked sigmoid tendency of the experimental data, which causes the value of $R^{2}=0.925$. But, as mentioned in the previous system, the Freundlich constant has a very high value $\left(K_{\mathrm{F}}=1,934,791,921\right.$ (mol of dye $/ \mathrm{mol}$ of Pec)(mol of dye/ $\mathrm{L})^{-1 / n}$, which means that this is unattainable when $\mathrm{Pec}$ is used to remove CR from an aqueous medium. Additionally, the value of the intensity constant $1 / n=1.152$ suggests that the adsorption is unlikely, contrasting with the high removal efficiency of CR by Pec found in Section 3.1.

Figure 4(c) shows the graphical form of Freundlich's linear equation for the Pec-MO-Ca system. In this figure, there is a great difference between the experimental data and the theoretical line that resulted from the Freundlich parameters reported in Table 2 for this system. Furthermore, the value of $K_{\mathrm{F}}=135,987,109$ (mol of dye/mol of $\mathrm{Pec}$ )( $\mathrm{mol}$ of dye/ $\mathrm{L})^{-1 / n}$ is very high, and it is unlikely that this system has such an extreme value. On the other hand, $1 / n=1.570$ indicates lower adsorption of MO to Pec than the DR80 and CR 
TABle 1: Parameters and goodness-of-fit measures from the linear form of the Langmuir model obtained by linear regression to experimental data from each Pec-dye-Ca system.

\begin{tabular}{|c|c|c|c|c|}
\hline & Pec-DR80-Ca & Pec-CR-Ca & Pec-MO-Ca & Pec-MR-Ca \\
\hline$K_{\mathrm{L}}(\mathrm{L} / \mathrm{mol}$ of dye $)$ & -91066.73 & -236581.17 & -3105.98 & 888.02 \\
\hline$q_{m}(\mathrm{~mol}$ of dye $/ \mathrm{mol}$ of $\mathrm{Pec})$ & -196.27 & -574.12 & -72.39 & 875.29 \\
\hline Intercept $1 /\left(K_{\mathrm{L}} \cdot q_{m}\right)$ & $5.59 E-08$ & $7.36 E-09$ & $4.45 E-06$ & $1.29 E-06$ \\
\hline Std. deviation of the intercept & $7.25 E-09$ & $2.00 E-09$ & $6.66 E-07$ & $1.83 E-07$ \\
\hline Slope $1 / q_{m}$ & $-5.09 E-03$ & $1.74 E-03$ & $1.38 E-02$ & $1.14 E-03$ \\
\hline Std. deviation of the slope & $2.16 E-03$ & $2.49 E-03$ & $3.51 E-03$ & $1.59 E-04$ \\
\hline$R^{2}$ & 0.271 & 0.032 & 0.509 & 0.776 \\
\hline Average relative error & 0.434 & 0.515 & 0.676 & 0.392 \\
\hline Std. deviation of the model & $2.21 E-08$ & $5.79 E-09$ & $1.41 E-06$ & $5.81 E-07$ \\
\hline
\end{tabular}

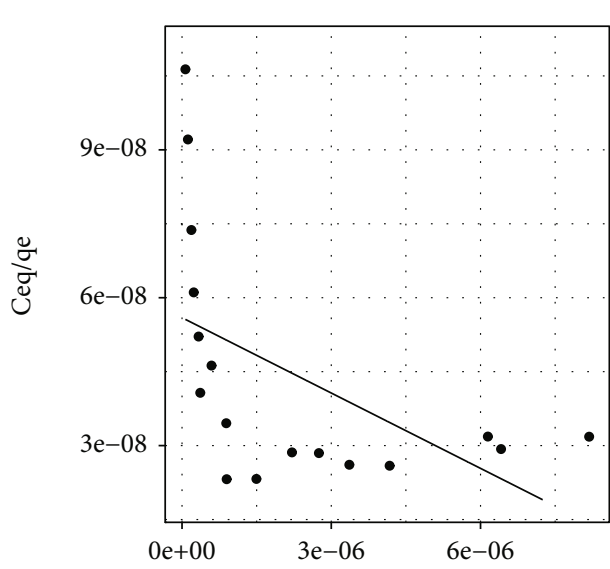

$\mathrm{Ceq}(\mathrm{mol} / \mathrm{L})$

(a)

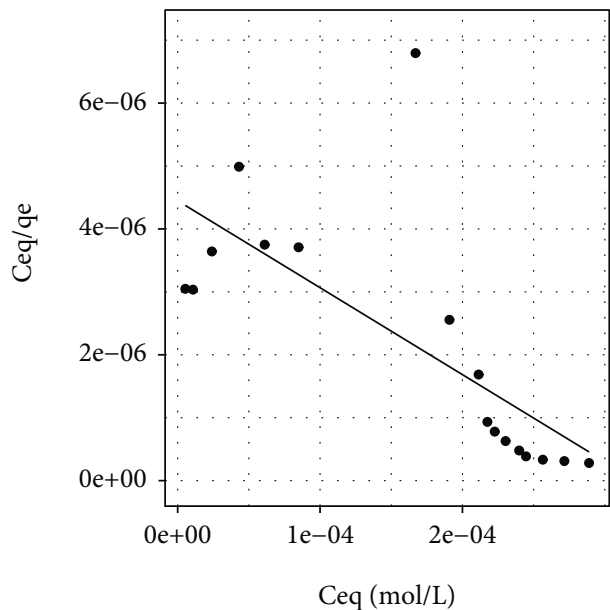

Ceq $(\mathrm{mol} / \mathrm{L})$

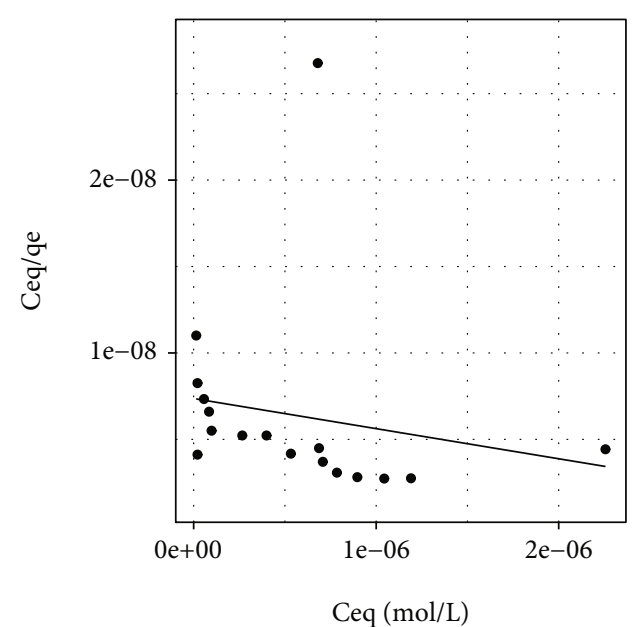

(b)

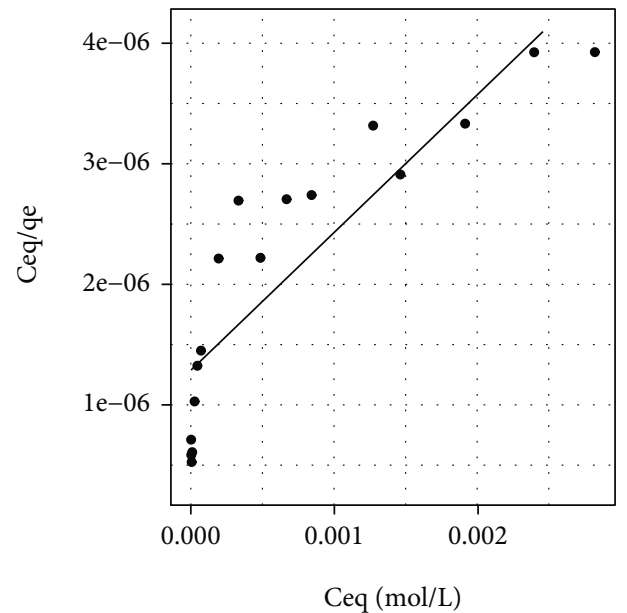

- Experimental data

Theoretical curve

(d)

FIgURE 3: Comparison of experimental data with the fitted linear form of the theoretical Langmuir isotherm for the following systems: (a) Pec-DR80-Ca, (b) Pec-CR-Ca, (c) Pec-MO-Ca, and (d) Pec-MR-Ca. 
TABle 2: Parameters and goodness-of-fit measures from the linear form of the Freundlich model obtained by linear regression to experimental data from each Pec-dye-Ca system.

\begin{tabular}{lcccc}
\hline & Pec-DR80-Ca & Pec-CR-Ca & Pec-MO-Ca & Pec-MR-Ca \\
\hline$K_{\mathrm{F}}(\mathrm{mol} \text { of dye/mol of Pec)(mol of dye/L) })^{-1 / n}$ & $1,127,755,625$ & $1,934,791,921$ & $135,987,109$ & 48,105 \\
$n$ & 0.785 & 0.868 & 0.637 & 1.393 \\
Intercept $\log \left(K_{\mathrm{F}}\right)$ & 9.052 & 9.287 & 8.133 & 4.682 \\
Std. deviation of the intercept & 0.263 & 0.562 & 0.714 & 0.065 \\
Slope $(1 / n)$ & 1.274 & 1.152 & 1.570 & 0.718 \\
Std. deviation of the slope & 0.044 & 0.085 & 0.178 & 0.016 \\
$R^{2}$ & 0.983 & 0.925 & 0.838 & 0.992 \\
Average relative error & 0.082 & 0.164 & 0.270 & 0.044 \\
Std. deviation of the model & 0.113 & 0.236 & 0.381 & 0.070 \\
\hline
\end{tabular}

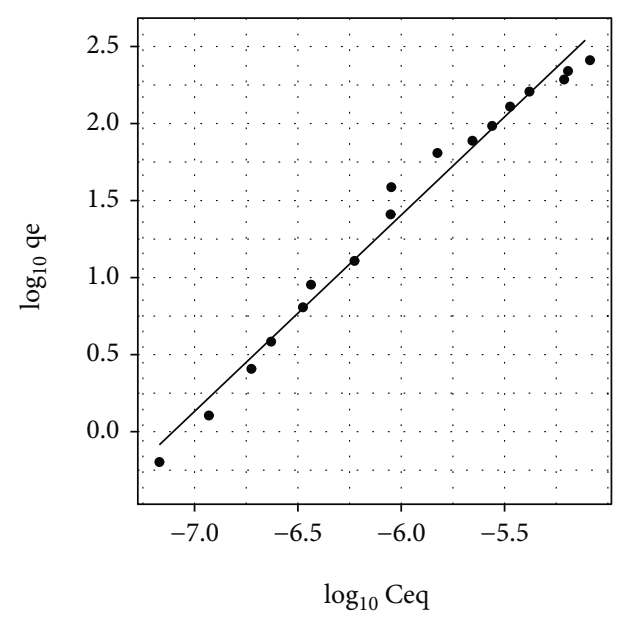

(a)

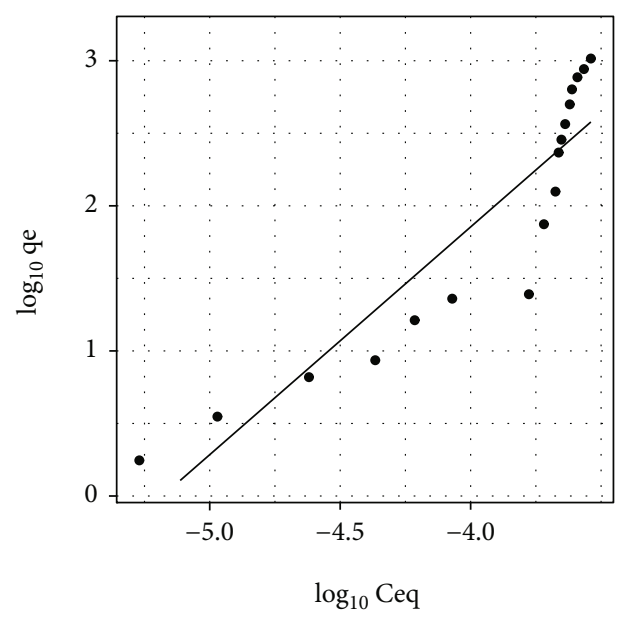

(c)

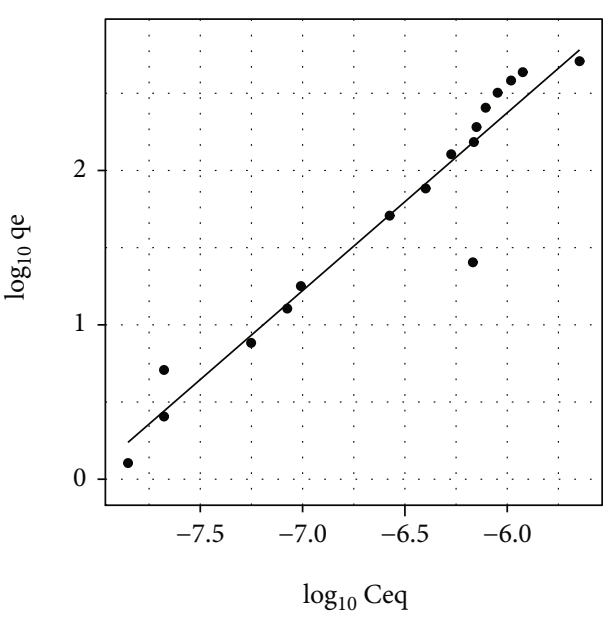

(b)

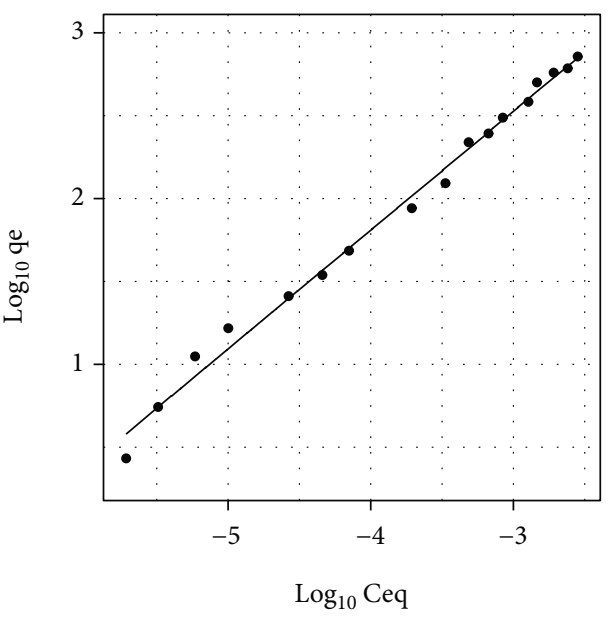

- Experimental data

Theoretical curve

(d)

FIgURE 4: Comparison of experimental data with the fitted linear form of the theoretical Freundlich isotherm for the following systems: (a) Pec-DR80-Ca, (b) Pec-CR-Ca, (c) Pec-MO-Ca, and (d) Pec-MR-Ca. 
dyes, which is true in qualitative terms but does not reliably describe what appears in this figure. Moreover, the value of $R^{2}=0.838$ indicates that the theoretical straight line fits poorly with the experimental isotherm.

Figure 4(d) shows that the Freundlich model fits better in the experimental data of the pec-MR-Ca system, which is demonstrated with a value of the coefficient of determination $R^{2}=0.992$. However, again a sigmoid shape is observed in the experimental isotherm. These findings suggest that MR has a certain tendency to form aggregates in this system, but at the same time, the experimental data can be described by this model. In this sense, it should be remembered that some characteristics of the Freundlich model are that this model considers the existence of regions with different affinities for the dye molecules, and as a consequence, there is a distribution of binding energies from the adsorption of the dye onto the adsorbent. In addition, there is no limitation of a dye monolayer on the surface of the Pec, and there may be interaction between the dye molecules when they adsorb to the Pec. These considerations originate from the values of $K_{\mathrm{F}}=48,105$ ( $\mathrm{mol}$ of dye $/ \mathrm{mol}$ of Pec)(mol of dye/ L) ${ }^{-1 / n}$, and $1 / n=0.718$ are feasible to have a physical meaning. However, as it will be seen later, the Zimm-Bragg model is also able to describe the experimental isotherm from PecMR-Ca and all of the other systems included in this study.

Some researchers have suggested that the poor fit of the Langmuir and Freundlich models is because polysaccharides such as xanthan and alginic acid can bind single molecules and molecular aggregates of dyes to their molecular surface. But at the same time, these polymers can form a threedimensional network by reacting with $\mathrm{Al}$ (III) and $\mathrm{Ca}$ (II) ions, respectively $[47,48]$. This polymerization process gives rise to a gel that traps all the "aggregated and nonaggregated dye molecules" adsorbed to the molecular surface of these biopolymers.

As mentioned before, the gelation mechanisms of alginic acid and Pec are very similar; then, it is suggested in this study that the Zimm-Bragg model could fit with the experimental data from the Pec-dye systems. This model has been used by some researchers to describe the isotherms obtained when some alkyl pyridinium- and alkyl trimethylammonium-based surfactant reacted with different polymers such as sodium dextran sulfate, sodium poly(styrene sulfonate), polyacrylate, (carboxymethyl) cellulose, polyacrylate, alginate, and pectate [49-52]. Moreover, they found a good agreement between theoretical curves obtained with this model and the experimental data.

The Zimm-Bragg model describes the formation of polymer-surfactant complexes since it considers that polymeric molecules can bind from a single surfactant molecule to molecular aggregates (micelles). Moreover, it was reported that the presence of different polymers in an aqueous phase favors the formation of micelles, which resulted in a decrease in their critical micellar concentration (CMC) [53]. In this sense, the mathematical description of the interaction phenomenon of the surfactant with the polymers is similar to the binding of some dye molecules and their aggregates to biopolymers, such as alginic acid and xanthan $[32,34,35,47,48]$. Moreover, to our knowledge, it has not been reported in any work where this model adequately describes the removal isotherms of azo dyes with native $\mathrm{Pec}$ and its derivatives.

In this model, it is considered that one dye molecule attached to the Pec molecular surface promotes the binding of other dye molecules remaining in the bulk solution. This trend is described by the constant $K_{u}$ or nucleation constant, which is obtained by the equation:

$$
K_{u}=\frac{B B}{(E B)(B)},
$$

where $B$ denotes one dye molecule adsorbed onto the Pec molecular surface, $B B$ indicates that two dye molecules attached to Pec occupy adjacent sites, and $E$ represents an empty site located at the molecular surface of this biopolymer. Similarly, the dye molecules that are already bounded to the Pec tend to form aggregates onto the molecular surface of this polysaccharide. This phenomenon is represented by the cooperativity constant $U$, which is determined by the following expression:

$$
U=\frac{(B B)(E E)}{(B E)^{2}} .
$$

These two parameters, $K_{u}$ and $U$, can be estimated by nonlinear regression, and a new variable named $\beta$ is obtained from $K_{u}$ and $U . \beta$ is defined as the ratio of Pec sites occupied by dye molecules divided by the total binding sites from this polymer. In the case where $\beta=1$, it indicates that all the sites from this polymer are occupied by dye molecules, and in counterpart $\beta=0$, it suggests that there is not any dye molecule adsorbed onto the Pec molecular surface. The distinct values of $\beta$ are calculated from the equation:

$$
\beta=\frac{1}{2}\left\{\frac{1+(s-1)}{\left[(1-s)^{2}+(4 s / U)\right]^{1 / 2}}\right\},
$$

where $s=K_{u} C_{\mathrm{eq}}$ and $C_{\mathrm{eq}}(\mathrm{mol} / \mathrm{L})$ was defined previously.

The theoretical parameters of the Zimm-Brag model can be obtained by fitting equation (9) to a set of experimental data. An empirical estimation of the $K_{u}$ parameter can be done by using the experimental data and finding the concentration $C_{\mathrm{eq}}$ value such as the $\beta$ value which is equal to 0.5 , as shown in equation (10). In a similar way, it is possible to empirically estimate the $U$ parameter by using equation (11). This empirical estimation can be useful for providing initial values in search algorithms designed to find the optimal values.

$$
\begin{gathered}
C_{\mathrm{eq}}(\beta=0.5)=\frac{1}{K_{u}}, \\
\frac{d \beta}{d \log C_{\mathrm{eq}}}(\beta=0.5)=\frac{U^{1 / 2}}{4} .
\end{gathered}
$$


In this work, the nonlinear regression procedure of the statistical software $\mathrm{R}$ [31] was used for estimating the $K_{u}$ and $U$ parameters. The nonlinear regression process allows determining the values of these parameters and provides statistical information, such as the standard deviation of the estimated parameters and the model.

Once the values of the parameters $K_{u}$ and $U$ have been estimated, the theoretical isotherm that describes the experimental values of $\beta$ is obtained.

Table 3 shows the values of the parameters of the ZimmBragg model for each Pec-Dye-Ca system, and Figure 5 depicts the experimental data and the theoretical isotherms that come from the substitution of $K_{u}$ and $U$ values in equation (9).

Interestingly, the trend in the $K_{u}$ values is as follows: $\mathrm{CR}>\mathrm{DR} 80>\mathrm{MO}>\mathrm{MR}$. This finding shows that CR has a higher tendency to form aggregates onto the Pec molecular surface than DR80. Moreover, $K_{u}$ values corresponding to $\mathrm{MO}$ and MR dyes notably have values smaller than those obtained in the Pec-CR-Ca and Pec-DR80-Ca systems. Lozano-Álvarez et al. [32] have proposed a relationship between structure and percentage removal when using alginic acid as a removal agent for azo dyes in the aqueous medium. In their study, they suggested that molecules with a higher positive charge, bigger size, and higher number of functional groups and aromatic rings would be those that would have a greater removal by alginic acid.

However, this "anomaly" encountered in the Pec-CR-Ca and Pec-DR80-Ca systems was also found in that report, indicating that the high ability of CR to form highly ordered aggregates and supramolecular structures was the principal phenomenon that explained the highest $K_{u}$ value observed for this dye. Similarly, the DR80 shows that its aggregation capacity is not as great as that shown by the CR, but it is large enough to obtain removal efficiency similar to that found in the CR (see the previous section).

On the other hand, the $K_{u}$ values of MO $(4,071.733)$ and MR (921.887) decrease drastically compared to the nucleation constants of the previous dyes. In this sense, Figure 2 shows that the size and molecular weight of MO are much smaller than those reported for DR80 and CR. In addition, the number of aromatic rings decreases dramatically in the MO and MR (2 phenyl rings), in comparison with four phenyl and two naphthyl rings attached to the DR80 and those included in CR (two naphthyl and two phenyl groups). The aggregation ability and affinity to Pec, which are mainly the result of the hydrogen bonding and hydrophobic interactions, are significantly reduced, as can be observed in the $K_{u}$ values included in Table 3 .

In the case of the cooperativity parameter $(U)$, the observed order is as follows: $\mathrm{MO}>\mathrm{CR}>\mathrm{DR} 80>\mathrm{MR}$. This finding indicates that $\mathrm{MO}$ molecules adsorbed to the Pec molecular surface have a very high ability to "migrate" and form aggregates onto Pec through several interactions (for example, hydrogen bonding, electrostatic, and hydrophobic interactions). However, given its small size, despite forming a considerable number of molecular aggregates onto the Pec polymer, in the conversion to the effective mass of removed dye, it was only possible to obtain an $88 \%$ removal efficiency that only exceeds that found in the Pec-MR-Ca system (62\%). The intermediate position of CR $(U=$ $13.783)$ and DR80 $(U=3.409)$ shows that these two dyes, once "fixed" to the molecular surface of Pec, have a low capacity to migrate due to supramolecular structures and the large aggregates that form CR and DR80, respectively. On the other hand, $\mathrm{Pec}$ is a hydrocolloid that when hydrated, it imparts a certain degree of turbidity that introduces a considerable level of uncertainty when each dye concentration is determined. This factor is included in the average relative error (ARE) parameter. However, the coefficient of determination corresponding to each system shows a good agreement between the theoretical isotherms with the experimental data.

From Table 3, it can be seen that the MR has the lowest $U$ value of all of the dyes $(U=2.513)$. Despite the structural similarity with $\mathrm{MO}$, the change of one sulfonate group by the carboxylate anion in the MR drastically decreases Pec binding capacity, especially in the hairy region, making it difficult to generate "nucleation centers" onto the Pec molecular surface. Although there may be the formation of aggregates between molecules adsorbed to $\mathrm{Pec}$, due to the lower number of MR molecules bound to Pec, the cooperativity parameter value is diminished. As mentioned above, the low tendency of MR to form aggregates explains the fact that the experimental isotherm of the Pec-MR-Ca system was the only one described adequately by the Freundlich and ZimmBragg models.

On the other hand, methylene blue (MB) and crystal violet $(\mathrm{CV})$ are dyes that have a high aggregation capacity [54], and they have been removed by different compounds derived from pectin. Li et al. [19] conducted studies of MB adsorption by calcium pectinate gel in powder form (PG) and found that of the models used, the Langmuir model was the one that best fits the experimental adsorption isotherm (they obtained a value of the coefficient of determination $R^{2}$ greater than that resulting from other models). However, the plot obtained from Freundlich's linear form $\left(\log q_{e}\right.$ versus $\log C_{\mathrm{eq}}$ ) shows that the experimental data follow a sigmoidal behavior instead of a straight line. This finding suggests that the molecules of this dye in the form of aggregates are binding to the PG. Furthermore, Yu et al. [55] synthesized pectin gel microspheres from calcium pectinate (PMP) and found again that the model with the highest $R^{2}$ value was the Langmuir model. However, the experimental data showed that in the graph from $\log q_{e}$ against $\log C_{\mathrm{eq}}$ (Freundlich's linear form), it does not fit with the straight line which was theoretically obtained. We propose that this difference between the experimental isotherm and the theoretical straight line obtained from the Freundlich linear model is due to the binding of MB molecules in the form of aggregates to the surface of the PMP.

Otherwise, Rakhshaee and Panahandeh [22] synthesized pectin grafted with carbodiimide and nanoparticles of $\mathrm{Fe}_{3}$ $\mathrm{O}_{4}$ bound to this polysaccharide (FN-PA). In addition, a second pectin derivative was obtained from the reaction of FN-PA with glutaraldehyde, and the material-denominated FN-PAG was produced. Finally, the last product resulted from the reaction between FN-PA with adipic acid, and this 
TABLe 3: Zimm-Bragg model parameters obtained by the nonlinear regression process to experimental data from each Pec-dye-Ca system.

\begin{tabular}{lcccc}
\hline & Pec-DR80-Ca & Pec-CR-Ca & Pec-MO-Ca & Pec-MR-Ca \\
\hline$K_{u}(\mathrm{~mol} / \mathrm{kg})$ & $258,892.994$ & $1,098,053.203$ & $4,071.733$ & 169.201 \\
$U$ & 3.409 & 13.783 & 12.799 & 2.513 \\
Std. deviation of $K_{u}$ & $7,481.307$ & $39,343.120$ & 15.136 & 38.015 \\
Std. deviation of $U$ & 0.423 & 4.299 & 0.995 & 0.389 \\
$R^{2}$ & 0.989 & 0.944 & 0.388 & 0.985 \\
Average relative error & 0.317 & 0.628 & 0.021 & 0.353 \\
Std. deviation of the model & 0.030 & 0.069 & 0.038 \\
\hline
\end{tabular}

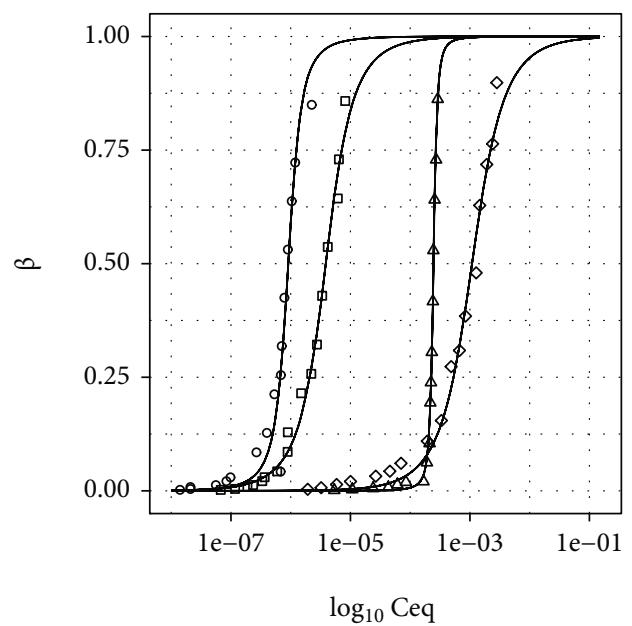

Figure 5: Application of the Zimm-Bragg model to the experimental data of the following systems: Pec-CR-Ca (open circles), Pec-DR80-Ca (open squares), Pec-MO-Ca (open triangles), and Pec-MR-Ca (open diamonds). The continuous lines represent the theoretical isotherms obtained from ZimmBragg parameters $K_{u}$ and $U$ from the different systems.

compound was abbreviated as FN-PAA. All of these compounds were used to remove $\mathrm{MB}$ from the aqueous phase. Interestingly, the grafting and binding of the nanoparticles of $\mathrm{Fe}_{3} \mathrm{O}_{4}$ to Pec favored the fit of the Langmuir model to the three experimental isotherms obtained with these three products. This finding suggests that grafting modified the steric configuration of the hairy region of Pec and the nanoparticles of $\mathrm{Fe}_{3} \mathrm{O}_{4}$ linked to $\mathrm{Pec}$ changed the ability of this biopolymer to induce the aggregation of $\mathrm{MB}$ molecules bound to the molecular surface of the FN-PA, FN-PAG, and FN-PAA.

In the same way, Lessa et al. [56] synthesized calcium pectate/cellulose microfiber beads ( $\mathrm{PB}-\mathrm{CF}$ ) to remove $\mathrm{MB}$ in the aqueous medium. This research group found that the Langmuir model fits adequately to the experimental data, which suggests that the cellulose microfibers embedded in the PB-CF particles modified the steric arrangement of the $\mathrm{Pec}$, and the porosity of the material that promoted it would bind the monomeric MB molecules instead of the molecular aggregates of this dye. Consequently, it was assumed that there was an MB monolayer on the surface of $\mathrm{PB}-\mathrm{CF}$ according to the fundamentals that support the Langmuir model.

On the other hand, Kodoth and Badalamoole [57] produced a nanocomposite obtained from the polymerization of 2-acrylamide-2-methyl-1-propane sulfonic acid (AMPS) and acrylamide (AAm) in the presence of N,N'-methylene-bisacrylamide (MBA), when mixing these compounds with a solution containing $\mathrm{Pec}$ with silver nanoparticles embedded in the molecular structure of this polysaccharide. This nanocomposite, which was abbreviated as Pec-g-poly (AMPS-co-AAm)/Ag, was used to adsorb CV in a concentration range between $20 \mathrm{mg} / \mathrm{L}$ and $2000 \mathrm{mg} / \mathrm{L}$, and it was found that the model that best fits the experimental isotherm was that of Langmuir. However, the experimental data included in the plots of the linear form of the Freundlich $\left(\log q_{e}\right.$ versus $\left.\log C_{\mathrm{eq}}\right)$ and Temkin $\left(q_{e}\right.$ versus $\left.\log C_{\mathrm{eq}}\right)$ models showed a sigmoid tendency, which suggests that the molecules of this dye were adsorbed to Pec-g-poly (AMPS-co-AAm)/Ag as molecular aggregates.

Otherwise, Mirza and Ahmad [58] synthesized an alginate/Pec nanocomposite, which was obtained from the reaction of the mixture of an aqueous solution of these two polysaccharides with glyoxal at $323 \mathrm{~K}$ for $24 \mathrm{~h}$. These reaction conditions favored the formation of acetal bonds between the hydroxyl groups of alginate and Pec with the keto group of glyoxal. In contrast to that described for Pec$g$-poly (AMPS-co-AAm)/Ag, the Langmuir model described adequately the experimental isotherm. Nevertheless, this concentration range was likely so small that the binding of aggregates of this dye to the alginate/Pec nanocomposite was not observed.

In summary, although there are reports of compounds based on Pec, the Zimm-Bragg model has not been used to describe the isotherms, probably due to the lack of perception of the aggregation phenomenon of the dyes that bind to the different Pec derivatives, and because the linear models of the Langmuir, Freundlich, and Temkin are generally used, the main adjustment parameter is the coefficient of determination $\left(R^{2}\right)$.

3.3. Visible Studies. In order to understand the interactions between Pec and dyes, several spectroscopic techniques such as visible, FT-IR, and Raman scattering were used.

Figure 6 shows the variation of the electronic absorption spectrum of DR80 $\left(1 * 10^{-5} \mathrm{M}\right)$ in an aqueous solution at 
distinct $\mathrm{pH}$ values at $\mathrm{IS}=0.1 \mathrm{M}$. From Figure 2(a), it can be seen that this dye contains several azo groups and has two naphthyl rings with one hydroxyl and azo groups attached to them in an ortho position. This structural configuration gives rise to a tautomeric azo-hydrazone equilibrium, and as a consequence, the peaks corresponding to these tautomers can be observed in the visible spectrum of DR80 [59].

It was reported that the azo form predominates in methanol [60], whose band is located at $\lambda=515 \mathrm{~nm}$, and the presence of both tautomers has been observed in dimethylsulfoxide (azo form at $\lambda=508 \mathrm{~nm}$ and hydrazone at $\lambda=540 \mathrm{~nm}$ ). However, in the aqueous medium, it was found that the maxima corresponding to the two tautomers coalesced, finding a single peak at $\lambda=530 \mathrm{~nm}$ at $\mathrm{pH}=4$, suggesting that the two forms (azo and hydrazone) remain in the aqueous environment.

As the $\mathrm{pH}$ increases, slight changes are observed in the intensity of the absorption maximum. Notably, Figure 6 shows that the original peak at $\mathrm{pH}=4(\lambda=530 \mathrm{~nm})$ gives rise to two bands at $\mathrm{pH}=12$, which correspond to the azo $(\lambda=515 \mathrm{~nm})$ and hydrazone $(\lambda=541 \mathrm{~nm})$ forms, which are deprotonated and originate from the common anion previously reported for dyes exhibiting this type of azohydrazone tautomerism [61].

In view of the fact that the highest removal value of DR80 using Pec was found at $\mathrm{pH}=9$ and $\mathrm{IS}=0.1 \mathrm{M}$ (see Section 3.1), the spectra of different dye solutions included in the range concentration between 5 and $200 \mathrm{mg} / \mathrm{L}$ were obtained at this $\mathrm{pH}$ value (Figure 7). From this figure, it can be seen that when the concentration of DR80 increases, the bands corresponding to the azo and hydrazone tautomers (located at $\lambda=510 \mathrm{~nm}$ and $\lambda=538 \mathrm{~nm}$, respectively) were identified.

It has been reported that in addition to the azohydrazone tautomerism, the formation of J-type aggregates also occurs in compounds that contain the hydroxyl group in ortho position to the azo group in aromatic compounds [62].

Figure 7 shows a band located at $\lambda=538 \mathrm{~nm}$, which suggests the presence of the hydrazone form in DR80, but at the same time, J aggregates were formed as a result of the formation of intermolecular hydrogen bonds between the sulfonate, hydrazone, and amide groups of this dye.

Interestingly, when DR80 reacts with pectin, a bigger difference is observed between the location of the characteristic peaks of the azo and hydrazone forms (located at $\lambda=$ $517 \mathrm{~nm}$ and $\lambda=544 \mathrm{~nm}$, respectively, for a dye concentration corresponding to $200 \mathrm{mg} / \mathrm{L}$, see Figure 8). This finding means that pectin interacts with DR80 more strongly than that observed with water molecules and favors the formation of J aggregates. As a consequence, this will result in dye binding to the neutral sugars of the hairy regions of the Pec through hydrogen bonds and hydrophobic interactions, despite the repulsion existing between negatively charged $\mathrm{DR} 80$ and $\mathrm{Pec}$ molecules at $\mathrm{pH}=9$.

In addition, it has been that the $\beta$-galactose residues of rhamnogalacturonan I (RG-I) purified from citrus Pec tend to self-aggregate forming through antiparallel pairing regions [63], which are potential sites to interact with

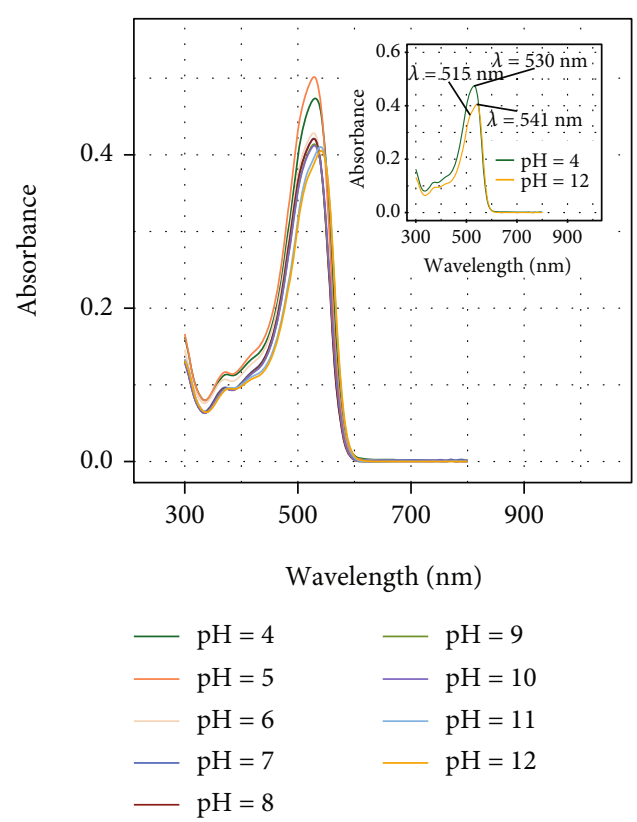

Figure 6: Visible absorption spectra of DR80 in aqueous solution at different $\mathrm{pH}$ values at $\mathrm{IS}=0.1 \mathrm{M}$.

DR80 through hydrogen bonding and hydrophobic interactions. In the same way, Perez et al. [64] have found that rhamnogalacturonan II (RG-II) tends to form dimers in vitro through a 1:2 borate-diol ester bond. These dimers contain neutral sugar residues that can also interact with DR80 in a similar manner to that mentioned for RG-I.

Figure 9 shows the visible absorption spectra of DR80 at different concentration values (50, 100, and $200 \mathrm{mg} / \mathrm{L}$ ) when the Pec-DR80-Ca product was formed. A bathochromic shift is again observed in the two absorption maxima corresponding to the azo and hydrazone forms, located at $\lambda=531 \mathrm{~nm}$ and $\lambda=559 \mathrm{~nm}$, respectively (at a concentration of $200 \mathrm{mg} /$ $\mathrm{L}$ of dye). These findings suggest that the formation of the calcium pectate (Pec-Ca) gel decreases the repulsion between DR80 and Pec, and as a consequence, the dye molecules bind to this polysaccharide to a greater extent through the same forces as they intervene in the Pec-DR80 adduct, but in a more intense way. Additionally, the insertion of the Ca (II) ions between the polygalacturonate chains causes the DR80 molecules to be closer. Moreover, an additional side-by-side aggregate formation of the DR80 molecules occurs due to the rearrangement in Pec molecules when Pec-DR80-Ca is formed [65].

Lozano-Alvarez et al. [35] have reported that the formation of calcium alginate (Alg-Ca) resulting from the reaction of sodium alginate with the calcium ions can be observed at the macroscopic level as a gel in which the dye molecules are "trapped," demonstrating the ability of this biopolymer in the removal of azo dyes. Braccini and Pérez [27] found that the formation of the Pec-Ca network is similar to that of calcium alginate. In this way, the phenomenon responsible for DR80 removal is an entrapment analogous to that reported for Alg-Ca [65]. 


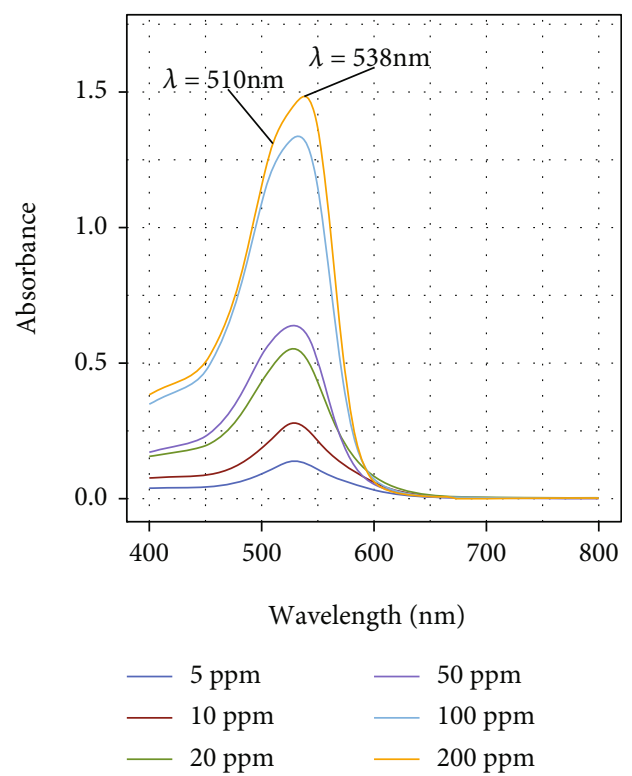

FIGURE 7: Visible absorption spectra of DR80 in aqueous solution at different concentration values $(\mathrm{pH}=9$ and $\mathrm{IS}=0.1 \mathrm{M})$.

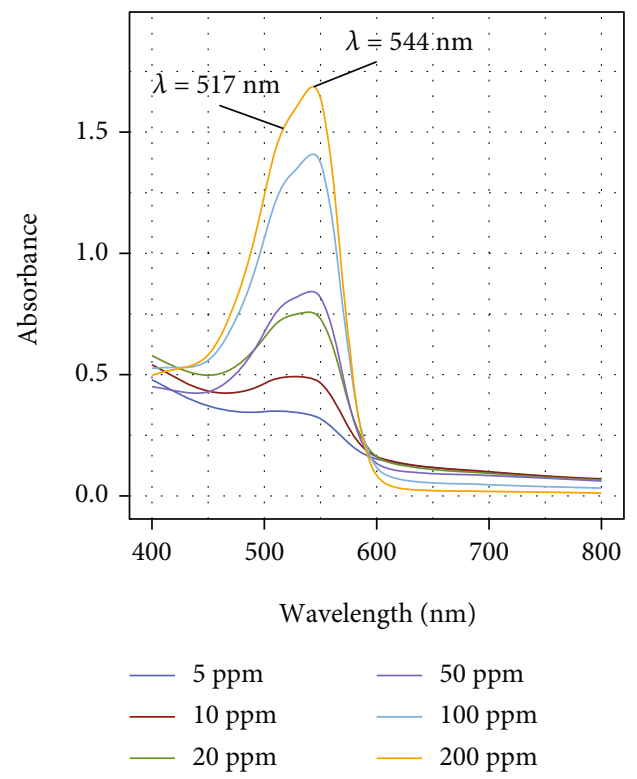

FIGURE 8: Visible absorption spectra of DR80 in aqueous solution at different concentration values in the presence of Pec $(1 \% \mathrm{w} / \mathrm{v}$, $\mathrm{pH}=9$, and $\mathrm{IS}=0.1 \mathrm{M}$ ).

Interestingly, the relative intensity of the azo and hydrazone forms is modified in the Pec-DR80-Ca, observing a slight increase in the band of the hydrazone form, suggesting that the chemical environment in which the DR80 molecules are encountered is slightly more polar concerning the aqueous medium [60].

It was reported that the visible spectrum of $\mathrm{CR}$ in an aqueous environment varies concerning the $\mathrm{pH}$ value of the aqueous phase [66]. For example, at neutral $\mathrm{pH}$ value, there are three absorption bands located at $\lambda=235 \mathrm{~nm}, \lambda=343$

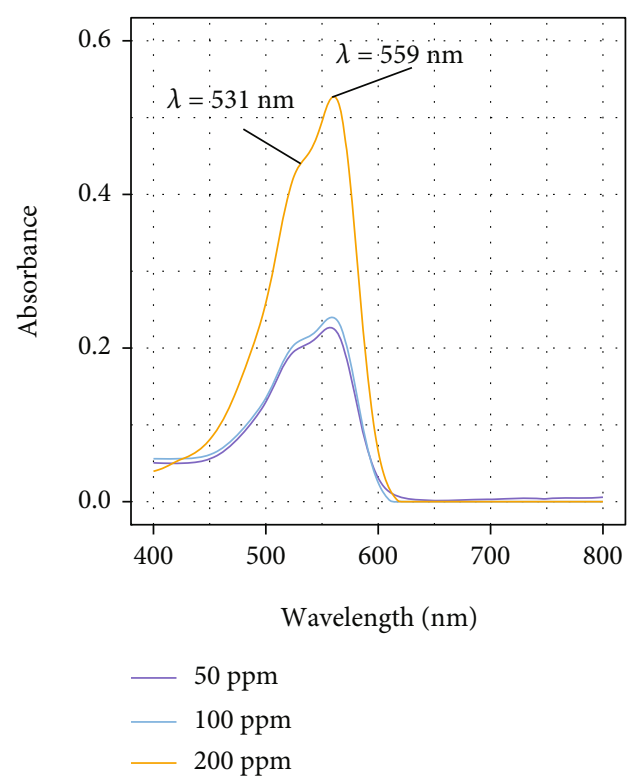

FIgURE 9: Visible absorption spectra of the Pec-DR80-Ca product at different dye concentration values: (a) $50 \mathrm{mg} / \mathrm{L}$, (b) $100 \mathrm{mg} / \mathrm{L}$, and (c) $200 \mathrm{mg} / \mathrm{L}$.

$\mathrm{nm}$, and $\lambda=496 \mathrm{~nm}$. These signals correspond to the transitions of the phenyl, naphthyl, and azo groups, respectively $[67,68]$. However, as the $\mathrm{pH}$ value is decreased, the location of the last band changes significantly, in such a way that at $\mathrm{pH}=4.0$, a blue-purple solution resulted from the protonation of the CR molecules. Several research groups have proposed a tautomeric equilibrium between the ammonium and quinoid forms $[69,70]$, as a result of the protonation of the nitrogen atoms of the amino and azo groups, respectively (see Figure 10).

An acid medium gives rise to a UV-visible spectrum where the bands located in the ultraviolet region decrease their intensity and the absorption maximum located at $\lambda=$ $520 \mathrm{~nm}$ appears, which was assigned to the ammonium form of CR. In the same way, it has been proposed that the azonium form of this dye can be detected like a shoulder between 600 and $800 \mathrm{~nm}$, whose intensity is affected by the rate of cooling [71]. In such a manner that when a solution of $\mathrm{CR}$ was recently prepared at acidic $\mathrm{pH}$ at room temperature, its spectrum shows only the characteristic peak located at $520 \mathrm{~nm}$, and the other bands are not clearly distinguished in the visible spectrum.

This finding coincides with the spectrum shown in Figure 11, where the characteristic peak of the ammonium form of $\mathrm{CR}$ is observed $\left(\lambda_{\max }=526 \mathrm{~nm}\right)$ and the signal assigned to the azonium form of the dye is not perceptible. In the same way, it was found that at low $\mathrm{pH}$ value, the $\mathrm{CR}$ molecules tend to form J aggregates [72]. This aggregation phenomenon causes a bathochromic shift of this band in the electronic spectrum of this dye, which agrees with the spectra shown in Figure 11. From this figure, it is observed that as the concentration of the dye is increased from 5 to $200 \mathrm{mg} / \mathrm{L}(\mathrm{pH}=4$ and $\mathrm{IS}=0.04 \mathrm{M})$, the absorption maximum is shifted from $\lambda=526 \mathrm{~nm}$ to $\lambda=545 \mathrm{~nm}$. 
<smiles>[CH2-][NH2+][PH+](C)C</smiles><smiles>[NH3+]C1C(NNC2CCC(C3CCC(NNC4CC(S(=O)(=O)O)C5CCCCC5C4[NH3+])CC3)CC2)CC(S(O)(O)O)C2CCCCC12</smiles><smiles>NNC1CCC(C2CCC([NH+]NC3C(N)CC(S(=O)(=O)[O-])C4CCCCC43)CC2)CC1</smiles>

FIgURE 10: Tautomeric forms of CR resulting from protonation at aqueous acidic solution: (a) deprotonated form, (b) ammonium form, and (c) quinoid or azonium form (adapted from Reference [71]).

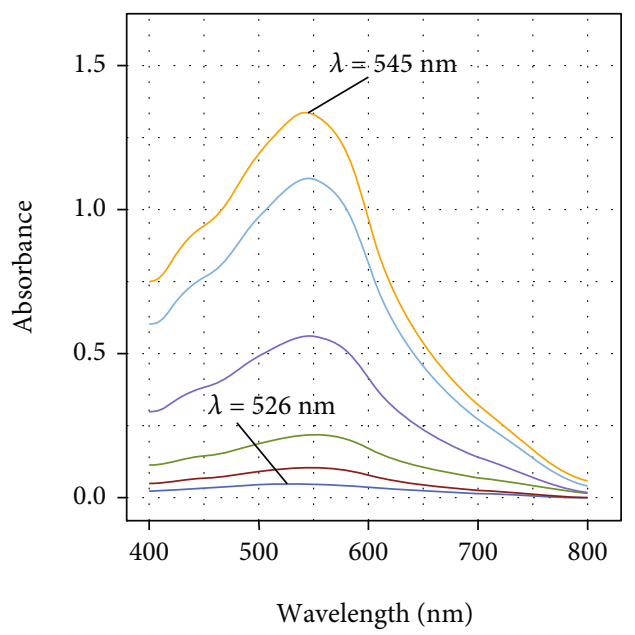

$$
\begin{array}{ll}
-5 \mathrm{ppm} & -50 \mathrm{ppm} \\
-10 \mathrm{ppm} & -100 \mathrm{ppm} \\
-20 \mathrm{ppm} & -200 \mathrm{ppm}
\end{array}
$$

FIGURE 11: Visible absorption spectra of CR in aqueous solution at different concentration values $(\mathrm{pH}=4$ and $\mathrm{IS}=0.04 \mathrm{M})$.

On the other hand, when Pec is added to CR solution ( $\mathrm{pH}=4$ and $\mathrm{IS}=0.04 \mathrm{M}$ ), a hypsochromic shift is dye concentration-dependent (see Figure 12). Thus, for example, a solution containing $5 \mathrm{mg} / \mathrm{L}$ of CR shows the absorption maximum at $\lambda=513 \mathrm{~nm}$, whereas a solution whose dye concentration is $200 \mathrm{mg} / \mathrm{L}$ shows this signal at $\lambda=505 \mathrm{~nm}$. These findings suggest that Pec promotes the formation of
$\mathrm{H}$ aggregates from this dye. This aggregation tendency occurs because the pKa of this dye (4.1) [38] is near to the $\mathrm{pH}$ value of the aqueous medium and consequently the CR molecules will have almost a net charge of zero. At the same time, at this $\mathrm{pH}$ value, there is a small repulsion among Pec and CR molecules, because the pKa value of this biopolymer is ranged between 2.8 and 4.1 [73]. As a result, the molecules of CR tend to form aggregates on the Pec molecular surface.

Furthermore, the formation of the molecular network promoted by the addition of the $\mathrm{Ca}^{+2}$ ions imposed a spatial constraint among CR molecules that decreased the extent of side-by-side dye aggregation, and the Coulombic attraction between the sulfonate groups of the CR molecules and the $\mathrm{Ca}^{+2}$ ions inserted in the Pec-Ca fragmented the $\mathrm{J}$ aggregates. This can be seen in Figure 13, where the absorption maxima are located at $\lambda_{\max }=505 \mathrm{~nm}$ at $50 \mathrm{mg} / \mathrm{L}$ and $\lambda_{\text {max }}=500 \mathrm{~nm}$ at $200 \mathrm{mg} / \mathrm{L}$.

The electronic absorption spectrum of $\mathrm{MO}$ has been previously reported by Tawara and Abu-Shamleh [74]. They detected a variation in the location of the absorption maxima when the $\mathrm{pH}$ of the solution of this dye was changed. Thus, when the $\mathrm{pH}=11$, the aqueous solution of the $\mathrm{MO}$ is yellow and shows a maximum at $\lambda=466 \mathrm{~nm}$. However, when the $\mathrm{pH}$ value decreases to $\mathrm{pH}=4$, this solution shows an orange color. This change is because there is a protonation in $\mathrm{MO}$ molecules whose $\mathrm{pKa}=3.37$ [75]. As was observed previously with $\mathrm{CR}$, when the MO molecules are protonated, they give rise to the tautomers azonium and ammonium at $\mathrm{pH}=4$ in an aqueous solution. The azonium form originates from the peak located at $\lambda=508 \mathrm{~nm}$, and the ammonium form will originate from the band located at $\lambda=316 \mathrm{~nm}$ from the UV-visible spectrum of MO.

The visible spectrum of this dye was obtained in the concentration range of $5-200 \mathrm{mg} / \mathrm{L}$ at $\mathrm{pH}=4$ and $\mathrm{IS}=$ $0.04 \mathrm{M}$, finding that the absorption maximum located at $\lambda_{\max }=467 \mathrm{~nm}$ remained at the same wavelength throughout this concentration range. The above suggests that $\mathrm{MO}$ has a low tendency to aggregate at this $\mathrm{pH}$ value. When Pec was added to MO aqueous solutions, a bathochromic shift of the absorption maximum was found and the new band appeared at $\lambda_{\max }=472 \mathrm{~nm}$, which remained constant despite the variation in the dye concentration $(5-200 \mathrm{mg} / \mathrm{L})$. This finding suggests that Pec favors the formation of J aggregates of MO molecules, which form as a consequence of the electrostatic interaction of the sulfonate group of one $\mathrm{MO}$ molecule with the dimethylamino group of another dye molecule, according to the results published by De Vylder and De Keukeleire [76]. In addition, this red shift is also attributed to the formation of hydrogen bonds between the azo groups of the $\mathrm{MO}$ and the hydroxyl groups of the sugar residues included in the Pec molecule [77, 78].

In the same way, it was observed that when the Pec-MOCa was formed, a new bathochromic shift of the absorption maximum occurred, and the new absorption maxima were located from $\lambda_{\max }=475 \mathrm{~nm}$ to $484 \mathrm{~nm}$ when dye concentration ranged from 5 to $200 \mathrm{mg} / \mathrm{L}$. This shift suggests that when the Pec-Ca network is formed as described by Braccini and Pérez [27], the intermolecular ionic interactions and the existing hydrogen bonding between $\mathrm{MO}$ and Pec mentioned 


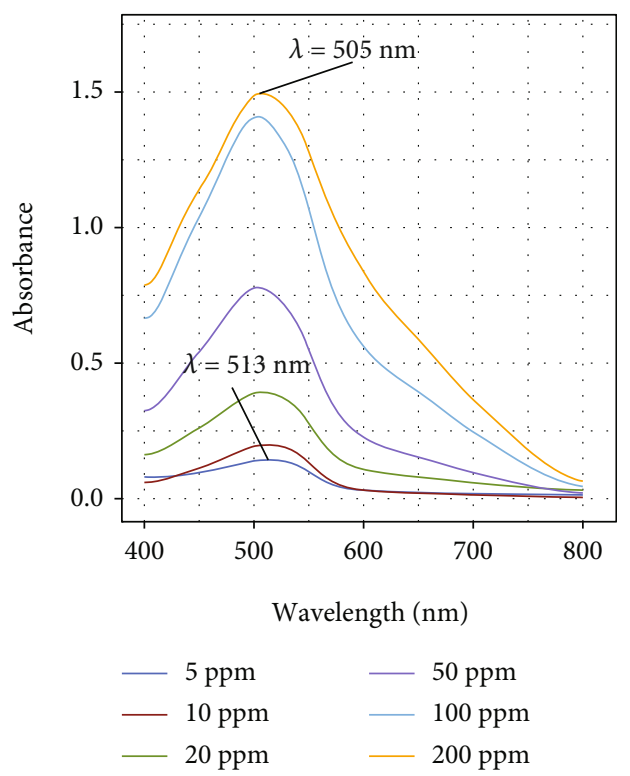

Figure 12: Visible absorption spectra of CR in aqueous solution at different concentration values in the presence of $\operatorname{Pec}(1 \% \mathrm{w} / \mathrm{v}$, $\mathrm{pH}=4$, and $\mathrm{IS}=0.04 \mathrm{M}$ ).

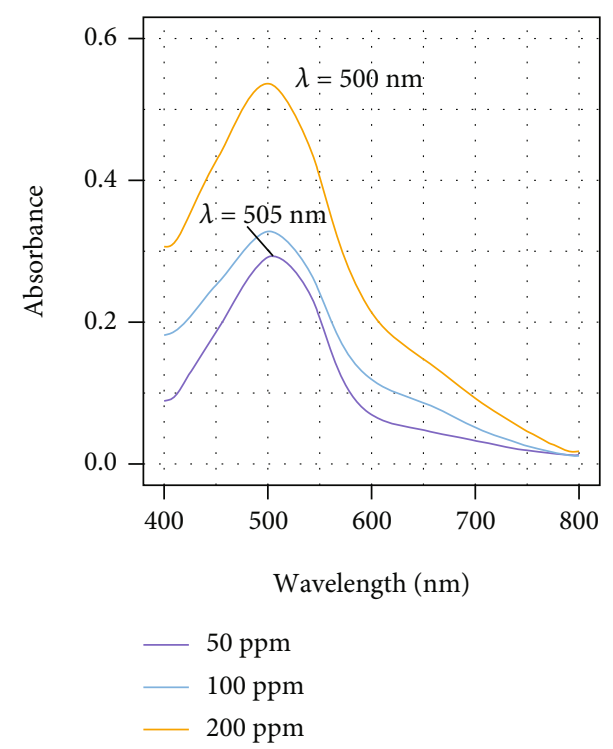

Figure 13: Visible absorption spectra of the Pec-CR-Ca product at different dye concentration values: $50 \mathrm{mg} / \mathrm{L}, 100 \mathrm{mg} / \mathrm{L}$, and $200 \mathrm{mg} / \mathrm{L}$.

above are reinforced, promoting the formation of more stable J aggregates.

On the other hand, Tawara and Abu-Shamleh have obtained the electronic absorption spectrum of MR at different $\mathrm{pH}$ values [79], and they found that MR has distinct UVvisible spectra when the $\mathrm{pH}$ value of its aqueous solution is changed. These researchers reported that in the $\mathrm{pH}$ range from 4 to 6 , the visible spectra of MR show considerable changes. This displacement in the absorption maxima is due to the presence of the anionic and monoprotonated spe- cies of this dye in an aqueous solution because its $\mathrm{pKa}=5.02$ [80]. It has been observed that at values lower than $\mathrm{pKa}$ (for example, $\mathrm{pH}=4$ ), the MR solution has a red color, which is caused by the presence of the monoprotonated species of $\mathrm{MR}$. In the same way that for $\mathrm{MO}$, at this $\mathrm{pH}$ value, the tautomeric forms azonium and ammonium can be found $[81,82]$. At neutral $\mathrm{pH}$, the aqueous solution containing this dye shows a yellow-orange color $(\lambda=430 \mathrm{~nm})$ that suggests the presence of deprotonated and protonated forms of MR [81]. In an alkaline medium, the absorption spectra of this dye are similar to those mentioned above, but with greater intensity than the spectra obtained at $\mathrm{pH}=7.0$.

In another way, measurements of electrical conductivity of MR solutions in an aqueous medium at $\mathrm{pH}=7.8$ suggest that the molecules of this dye are in monomeric form in the concentration range from $1 * 10^{-4} \mathrm{M}(26.93 \mathrm{mg} / \mathrm{L})$ to 9.998 $* 10^{-4} \mathrm{M}(268.9 \mathrm{mg} / \mathrm{L})$ [83]. In agreement with the above, we found that the electron absorption spectra of the MR at $\mathrm{pH}=7$ and $\mathrm{IS}=0.04 \mathrm{M}$ using different concentration values $(5-200 \mathrm{mg} / \mathrm{L})$ show a peak located at $\lambda=430 \mathrm{~nm}$ (deprotonated form) and a shoulder centered at $\lambda=555 \mathrm{~nm}$ corresponding to the presence of the monoprotonated form of this dye.

These experimental results suggest that under these conditions, MR does not tend to aggregate. In the same way, when Pec was added to the MR in an aqueous solution at $\mathrm{pH}=7$ and $\mathrm{IS}=0.04 \mathrm{M}$, it was found that the location of the absorption maximum and the shoulder located at $\lambda=$ $430 \mathrm{~nm}$ and $\lambda=555 \mathrm{~nm}$, respectively, did not change, indicating that the molecules of this dye are in monomeric form in the presence and the absence of Pec. This finding suggests a certain degree of repulsion between the Pec and MR molecules and that the main forces involved in the adsorption of this dye onto the molecular surface of the Pec are hydrogen bonds and hydrophobic interactions [84], but more weakly than those existing in the Pec-MO system. Furthermore, although ionic interaction between the dimethylamino group of the MR with the carboxylate groups of the galacturonate residues of the Pec molecules exists, it is not capable of changing the electronic distribution of the MR molecules.

When the Pec-MR-Ca was formed, a bathochromic shift was observed, and the new absorption maximum ranged from $\lambda_{\max }=432 \mathrm{~nm}$ to $\lambda_{\max }=445 \mathrm{~nm}$ at a dye concentration interval from 5 to $200 \mathrm{mg} / \mathrm{L}$ in the visible spectrum of this dye. However, the shoulder of the monoprotonated form remained at $\lambda=445 \mathrm{~nm}$ at any concentration of the MR. Lozano-Álvarez et al. [32] reported that sodium alginate interacted with the MR but did not modify its $\lambda_{\max }$ value. Furthermore, the alginate-MR-Ca product did not change the location of the absorption maximum of the visible spectrum of this dye. Pec has a polygalacturonate backbone similar to the structure of alginate that contains regions of polymannuronate and polyguluronate and mixed regions of these glucuronic acids, which impart a negative charge to the alginate. As mentioned before, unlike alginate, Pec has a hairy zone, which contains residues of neutral sugars that promote the reduction of repulsion between Pec and MR when gelling occurs after the addition of the $\mathrm{Ca}^{+2}$ ion. Consequently, dye molecules interact with the rhamnogalacturonan I (RG-I) 
and rhamnogalacturonan II (RG-II) segments of Pec through the formation of hydrogen bonding and hydrophobic interactions, favoring the formation of J aggregates.

3.4. Infrared and Raman Studies. To understand in greater detail the interactions among Pec and the dyes, FT-IR and Raman spectra of the following compounds were obtained: dye, Pec-Ca, and Pec-dye-Ca. The last two compounds were produced using the same procedure used in the removal isotherms (see Section 2.3 of the methodology). Thus, Figures 12(a)-12(c) show the FT-IR spectra of DR80, Pec$\mathrm{Ca}$, and Pec-DR80-Ca, respectively.

In Figure 14(a), the signal located at $3390 \mathrm{~cm}^{-1}$ corresponds to the vibration ${ }_{v}(\mathrm{OH})$ of the hydroxyl groups attached to the DR80 molecules, and the band assigned to the vibration ${ }_{v}(\mathrm{NH})$ of the substituted carbamoylamino group located at $3100 \mathrm{~cm}^{-1}$ was found to form hydrogen bonds. These two signals prevent a clear observation of vibration ${ }_{v}(\mathrm{CH})$ from the phenyl and naphthyl groups of the dye.

The signals corresponding to the vibrations ${ }_{v}(\mathrm{C}=\mathrm{O})$ and ${ }_{v}(\mathrm{C}=\mathrm{N})$ located at $1647 \mathrm{~cm}^{-1}$ and $1565 \mathrm{~cm}^{-1}$, respectively, suggest the presence of the hydrazone form in DR80 [85]. Additionally, the signal ${ }_{\delta}(\mathrm{N}-\mathrm{H})$ in the bending plane of the substituted carbamoylamino group located at $1479 \mathrm{~cm}^{-1}$ can be identified [86].

Figures 12(b) and 12(c) correspond to the spectra of Pec-Ca and Pec-DR80-Ca compounds. As can be seen, they are very similar, and consequently, the signals previously described for the DR80 cannot be easily identified in Figure 14(c). In this sense, the characteristic peak of the hydroxyl group attached to the DR80 molecule is immersed in the typical vibration of the alcohol groups of the sugar residues belonging to Pec-Ca.

This signal is centered at $3240 \mathrm{~cm}^{-1}$ and ranges from $3600 \mathrm{~cm}^{-1}$ to $2900 \mathrm{~cm}^{-1}$ in both compounds, Pec-Ca and Pec-DR80-Ca. Since the ${ }_{v}$ asym(COO) and ${ }_{v}$ sym(COO) signals located at $1583 \mathrm{~cm}^{-1}$ and $1422 \mathrm{~cm}^{-1}$ vibrate at the same frequency in Pec-Ca and Pec-DR80-Ca, they suggest that the network proposed by Braccini and Pérez [27] for Pec-Ca is very similar in both compounds.

Unfortunately, these two peaks from the carboxylate group in its calcium salt form avoid the identification of the bands assigned to the vibrations ${ }_{v}(\mathrm{C}=\mathrm{O})$ and ${ }_{v}(\mathrm{C}=\mathrm{N})$ found in the FT-IR spectrum of the dye in the solid state. On the other hand, the signal located at $1484 \mathrm{~cm}^{-1}$ of the bending ${ }_{\delta}(\mathrm{N}-\mathrm{H})$ in the plane found in the FT-IR spectrum of Pec-DR80-Ca confirms the presence of DR80 in this compound.

Figure 15 shows the Raman spectra of DR80, PecDR80-Ca, and Pec-Ca. In this figure, it is observed that Pec-Ca does not have vibrations that result from the Raman effect (see Figure 15(c)), and the signals observed in the Raman spectrum of Pec-DR80-Ca are due to the presence of DR80 in this compound. In this sense, the vibrations ${ }_{v}(\mathrm{C}=\mathrm{C})$ from phenyl and naphthyl groups attached to the DR80 molecule are located at $1594 \mathrm{~cm}^{-1}$ and $1259 \mathrm{~cm}^{-1}$, respectively.

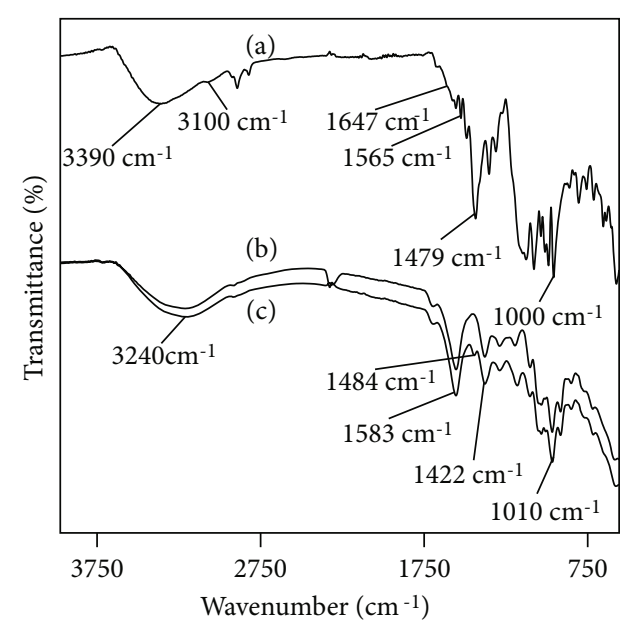

FIGURE 14: FT-IR spectra of the following compounds: (a) DR80, (b) Pec-Ca, and (c) Pec-DR80-Ca.

Figure 15(b) shows that these signals are found at $1594 \mathrm{~cm}^{-1}$ and $1267 \mathrm{~cm}^{-1}$ in the Pec-DR80-Ca product, suggesting that the aromatic rings of the naphthyl groups in this last compound "slightly changed" their chemical environment, which is consistent with the visible spectroscopy studies.

The vibration ${ }_{v}(\mathrm{C}=\mathrm{N})$ of the hydrazone tautomer is located at $1565 \mathrm{~cm}^{-1}$ in the spectrum of the dye. This same vibration appears at $1566 \mathrm{~cm}^{-1}$ in the Pec-DR80-Ca spectrum, suggesting that this dye is present in the hydrazone form in this compound. The peaks that vibrate at $1174 \mathrm{~cm}^{-1}$ and $1154 \mathrm{~cm}^{-1}$ correspond to the vibration mode ${ }_{v}($ phenyl- $\mathrm{N})=\mathrm{N}$ detected in the DR80 spectrum.

Interestingly, these peaks are found in the Pec-DR80-Ca spectrum (signals located at $1177 \mathrm{~cm}^{-1}$ and $1154 \mathrm{~cm}^{-1}$ ), suggesting that the bond formed by the $\alpha$-nitrogen atom from the azo group and the carbon atom from the phenolic ring has a bonding order close to one $[82,87]$. Therefore, it suggests that the azo group has a bonding order corresponding to two. This proposal was confirmed with the peaks located at $1448 \mathrm{~cm}^{-1}$ and $1450 \mathrm{~cm}^{-1}$ in the spectra of DR80 and Pec-DR80-Ca, respectively, which were assigned to the vibration ${ }_{v}(\mathrm{~N}=\mathrm{N})$ of the azo group $[88,89]$. These findings indicate that the hydrazone and azo tautomers found in the dye remain in the Pec-DR80-Ca.

The FT-IR spectrum of the CR shows the signals $v$ $\operatorname{asym}(\mathrm{NH})$ and ${ }_{\nu} \operatorname{sym}(\mathrm{NH})$ located at $3460 \mathrm{~cm}^{-1}$ and 3350 $\mathrm{cm}^{-1}$, respectively, which are typical of an aromatic amine (see Figure 2(b)), which in this case correspond to the amino group attached to the naphthyl group. Additionally, the existence of the amino group is confirmed with the vibration ${ }_{v}(\mathrm{C}-\mathrm{N})$ found at $1346 \mathrm{~cm}^{-1}$ and the signal ${ }_{\delta}(\mathrm{N}-\mathrm{H})$ in the bending plane located at $1583 \mathrm{~cm}^{-1}$. Also, the vibration ${ }_{v}(\mathrm{C}-\mathrm{H})$ of aromatic rings located at $3075 \mathrm{~cm}^{-1}$ suggests the presence of the phenyl and naphthyl groups.

On the other hand, a broad band corresponding to the vibration ${ }_{v}(\mathrm{OH})$ centered at $3250 \mathrm{~cm}^{-1}$ and $3280 \mathrm{~cm}^{-1}$ in the spectra of Pec-Ca and Pec-CR-Ca, respectively, avoided 


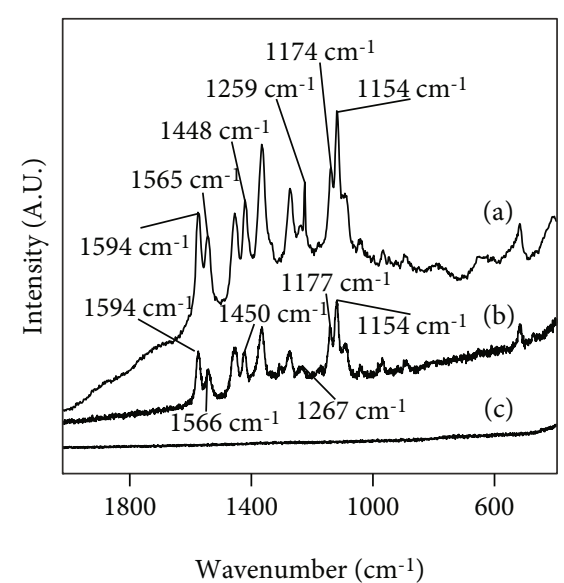

FIGURE 15: Raman spectra of the following compounds: (a) DR80, (b) Pec-DR80-Ca, and (c) Pec-Ca.

the identification of typical vibrations of the amino group and those signals from the phenyl and naphthyl groups attached to the CR molecule. The region of the carbonyl group is very similar in Pec-Ca and Pec-CR-Ca, in such a manner that the signal ${ }_{v}(\mathrm{CO})$ from the methyl ester found at $1743 \mathrm{~cm}^{-1}$ and $1740 \mathrm{~cm}^{-1}$, respectively, showed a small intensity, indicating a low degree of methoxylation in both compounds. Similarly, the vibrations $v_{v}$ asym(COO) and ${ }_{v}$ $\operatorname{sym}(\mathrm{COO})$ were located at $1600 \mathrm{~cm}^{-1}$ and $1424 \mathrm{~cm}^{-1}$, respectively, in the FT-IR spectrum from Pec-Ca and at $1594 \mathrm{~cm}^{-1}$ and $1420 \mathrm{~cm}^{-1}$ in the case of Pec-CR-Ca, suggesting that the polymeric structure formed in Pec-Ca by the polygalacturonate regions does not undergo considerable structural changes in Pec-CR-Ca due to the presence of dye.

The signal corresponding to the bending ${ }_{\delta}(\mathrm{NH})$, which is located at a lower frequency $\left(1512 \mathrm{~cm}^{-1}\right)$ in the Pec-CR$\mathrm{Ca}$ than the signal observed in the spectrum of the dye in the solid-state $\left(1583 \mathrm{~cm}^{-1}\right)$, suggests that the amino groups are protonated. The above is the consequence of the acidic $\mathrm{pH}$ of the aqueous medium in which $\mathrm{CR}$ and Pec react and indicate that dye molecules are in the ammonium form [90].

The ${ }_{v}(\mathrm{C}=\mathrm{C})$ vibrations of the phenyl and naphthyl groups appeared in the Raman spectrum of $\mathrm{CR}$ at $1590 \mathrm{~cm}^{-1}$ and $1357 \mathrm{~cm}^{-1}$, respectively [87]. These same signals are located at $1590 \mathrm{~cm}^{-1}$ and $1367 \mathrm{~cm}^{-1}$ in the Raman spectrum from Pec-CR-Ca, confirming the presence of $\mathrm{CR}$ in this last compound. In the same way, the vibration ${ }_{v}(\mathrm{~N}=\mathrm{N})$ from the azo group encountered at $1450 \mathrm{~cm}^{-1}$ in the Raman spectra of CR and Pec-CR-Ca suggests that the ammonium tautomer is present in both compounds.

On the other hand, the vibration ${ }_{\nu}(\mathrm{C}-\mathrm{N})$, where the carbon atom is part of the naphthyl groups of CR, originates from two peaks located at $1154 \mathrm{~cm}^{-1}$ and $1132 \mathrm{~cm}^{-1}$. Interestingly, in the Pec-CR-Ca, these signals move to $1177 \mathrm{~cm}^{-1}$ and $1157 \mathrm{~cm}^{-1}$, suggesting an increase in the order of the C$\mathrm{N}$ bond. And consequently, it is deduced that the azonium form is in a higher proportion than the dye in a solid state (see Figure 10) [90]. These findings indicate that the two tautomers (ammonium and azonium) are present in the Pec-CR-Ca.

The FT-IR spectrum of MO shows the broad band assigned to the vibration ${ }_{v}(\mathrm{~N}-\mathrm{H})$, which is centered at $3440 \mathrm{~cm}^{-1}$ indicating that the dimethylamino group is protonated and that it is forming hydrogen bonds in its powder form [86]. The vibrations ${ }_{v}$ asym $(\mathrm{C}-\mathrm{H})$ and ${ }_{v} \operatorname{sym}(\mathrm{C}-\mathrm{H})$ of the methyl groups appear at $2911 \mathrm{~cm}^{-1}$ and $2803 \mathrm{~cm}^{-1}$, respectively. The vibration ${ }_{v}(\mathrm{C}-\mathrm{C})$ from the phenyl and naphthyl groups appears at $1601 \mathrm{~cm}^{-1}$, and the signal ${ }_{v}(\mathrm{C}$ $\mathrm{N}$ ), whose vibration frequency is $1366 \mathrm{~cm}^{-1}$, suggests the presence of an aromatic amine in the $\mathrm{MO}$ molecules. On the other hand, the FT-IR spectra of Pec-Ca and Pec-MO$\mathrm{Ca}$ seem to be very similar. As was described before for the previous dyes, this finding makes it difficult to identify the peaks coming from the dye in the Pec-MO-Ca product. For example, the broad band centered at $3288 \mathrm{~cm}^{-1}$ corresponding to the vibration ${ }_{v}(\mathrm{O}-\mathrm{H})$ in the Pec-MO$\mathrm{Ca}$ spectrum prevents the detection of the ${ }_{v}(\mathrm{~N}-\mathrm{H})$ signal from the dimethylamino group attached to MO molecules. A small peak was found at $1739 \mathrm{~cm}^{-1}$, indicating that some galacturonate residues are methoxylated. The signals that correspond to the vibrations $v_{v} \operatorname{asym}(\mathrm{COO})$ and $v$ $\operatorname{sym}(\mathrm{COO})$ appear at $1600 \mathrm{~cm}^{-1}$ and $1416 \mathrm{~cm}^{-1}$, respectively, in the Pec-Ca, which are located at $1597 \mathrm{~cm}^{-1}$ and $1415 \mathrm{~cm}^{-1}$ in the Pec-MO-Ca, indicating that the carboxyl group of the galacturonate residues is not directly involved in the removal of MO. The only peak that confirms the presence of MO in the Pec-MO-Ca is found at $1361 \mathrm{~cm}^{-1}$ and corresponds to the vibration ${ }_{v}(\mathrm{C}-\mathrm{N})$ of an aromatic amine (see Figure $\left.2(\mathrm{c})\right)$. In contrast to FT-IR data, the Raman spectrum of Pec-MO-Ca irrefutably confirms that $\mathrm{MO}$ is present in this compound. Thus, the bands assigned to the vibrations ${ }_{v}(\mathrm{C}=\mathrm{C})$ and ${ }_{v}$ $(\mathrm{N}=\mathrm{N})$ of the phenyl group and azo group are located at $1588 \mathrm{~cm}^{-1}$ and $1443 \mathrm{~cm}^{-1}$, respectively, in the spectrum of MO. These same signals appear at $1589 \mathrm{~cm}^{-1}$ and $1441 \mathrm{~cm}^{-1}$ in the spectrum of Pec-MO-Ca. Lee et al. [91] reported that the vibration of the protonated azo group ${ }_{v}(-\mathrm{N}-\mathrm{N}-\mathrm{H})$ appears at $1411 \mathrm{~cm}^{-1}$. The Raman spectrum of MO shows this peak at $1410 \mathrm{~cm}^{-1}$, and in the spectrum of Pec-MO-Ca, it is located at $1414 \mathrm{~cm}^{-1}$, indicating that a fraction of this dye remains in the azonium form in both compounds (MO and Pec-MO-Ca).

In the FT-IR spectrum of the MR, it is possible to identify the signal corresponding to the vibration ${ }_{v}(\mathrm{C}-\mathrm{H})$ located at $3050 \mathrm{~cm}^{-1}$, which is typical of aromatic rings. The characteristic bands of the carboxylate group attached to this dye in its sodium salt, ${ }_{v}$ asym $(\mathrm{COO})$ and ${ }_{v}$ sym $(\mathrm{COO})$, are located at $1595 \mathrm{~cm}^{-1}$ and $1441 \mathrm{~cm}^{-1}$, respectively. Additionally, the vibration ${ }_{v}(\mathrm{C}-\mathrm{N})$ of an aromatic amine appears at $1363 \mathrm{~cm}^{-1}$. In the same way as that found in the Pec-MO$\mathrm{Ca}$ system, the FT-IR spectra of Pec-Ca and Pec-MR-Ca are very similar. In such a way, the broad band of the vibration ${ }_{v}(\mathrm{OH})$ centered at $3251 \mathrm{~cm}^{-1}$ from this compound prevents the detection and identification of MR signals included in the frequency range from 3200 to $2600 \mathrm{~cm}^{-1}$. Due to the neutral $\mathrm{pH}$ in which the Pec-MR-Ca was obtained, it can be concluded that the hydrolysis of the methyl ester was incomplete because a small peak located at $1740 \mathrm{~cm}^{-1}$ appears in 
the FT-IR spectrum of this dye. The typical signals of the vibrations ${ }_{v}$ asym $(\mathrm{COO})$ and ${ }_{v}$ sym(COO) located at $1598 \mathrm{~cm}^{-1}$ and $1405 \mathrm{~cm}^{-1}$ coming from the galacturonate residues in Pec-MR-Ca prevented the detection of the carboxylate group attached to MR molecules trapped in this product. Unfortunately, the ${ }_{v}(\mathrm{C}-\mathrm{N})$ signal of the aromatic amine of the MR was not identified because it was immersed in the signals included in the FT-IR of Pec-MR-Ca.

On the other hand, the Raman spectrum of Pec-MRCa contains the signals ${ }_{v}(\mathrm{C}=\mathrm{C})$ from phenyl rings and ${ }_{v}$ $(\mathrm{N}=\mathrm{N})$ of the azo group located at $1588 \mathrm{~cm}^{-1}$ and $1434 \mathrm{~cm}^{-1}$, respectively. Two peaks with similar frequency $\left(1589 \mathrm{~cm}^{-1}\right.$ and $1443 \mathrm{~cm}^{-1}$, respectively) appeared in the Raman spectrum of MR, confirming the presence of this dye in Pec-MR-Ca.

\section{Conclusions}

The use of Pec to remove the azo dyes DR80, CR, MO, and MR resulted in the following removal percentages under optimal pH and IS conditions: DR80 (99.5\%), CR (99.8\%), MO (88.6\%), and MR (68\%).

The reaction of Pec with the $\mathrm{Ca}^{+2}$ ion produced Pec-Ca, which formed a polymeric network that "traps" the dye molecules adsorbed to this polysaccharide, removing them from an aqueous solution.

To our knowledge, this is the first time that the ZimmBragg model is applied to explain the mechanism for the removal of azo dyes with native Pec. This model adequately describes the experimental isotherms because it considers that Pec not only can bind to the dye molecules in their monomeric form but also adsorb and trap aggregates of dye. In this sense, it is notable that the experimental isotherms of the dyes with a high aggregation ability were best described by the Zimm-Bragg model. However, when the aggregation capacity of the dye molecules is very low, the Freundlich model can describe the experimental data.

The values of the nucleation constant $K_{u}$ were notably higher in DR80 and CR than in MO and MR, suggesting that large dye molecules, with a high number of big aromatic groups, with functional groups capable of forming ionic bonds, hydrogen bonds, and hydrophobic interactions, result in high values of this parameter.

The migration ability of the dyes onto the molecular surface of the Pec, with the consequent formation of dimers, trimers, and multimers, is included in the aggregation constant $U$. This parameter followed the order $\mathrm{MO}>\mathrm{CR}>\mathrm{DR} 80>\mathrm{MR}$, indicating that dye molecules adsorbed to Pec with high mobility onto the molecular surface of this polysaccharide yielded the highest values of this parameter. In counterpart, dyes having a low affinity for Pec and a limited tendency to aggregate after being adsorbed onto this biopolymer yielded small values of the $U$ parameter.

In addition, the biological degradation of dyes in the Pec-Dye-Ca residue is being studied, obtaining promising results, which suggests that since Pec is a biodegradable biopolymer, its use in the removal of azo dyes is a sustainable biotechnological option for the treatment of effluents contaminated with these compounds.

\section{Data Availability}

To request data about direct red 80 , Congo red, methyl orange, and methyl red, contact Juan Antonio LozanoÁlvarez via the email lozanoalvarez@yahoo.com.

\section{Conflicts of Interest}

The authors declare that they have no conflicts of interest.

\section{Acknowledgments}

This work was supported by the Universidad Autónoma de Aguascalientes through grants PIBT19-3 and PIM21-4.

\section{Supplementary Materials}

A graphical abstract is provided as supplementary material. (Supplementary Materials)

\section{References}

[1] J. A. Kent, "Dye application, manufacture of dye intermediates and dyes," in Handbook of Industrial Chemistry and Biotechnology, vol. 1, pp. 475-548, Springer, 2012.

[2] M. Clark, Handbook of Textile and Industrial Dyeing, Woodhead Publishing Series in Textiles, 2011.

[3] H. Zheng, J. Zhang, J. Yan, and L. Zheng, "An industrial scale multiple supercritical carbon dioxide apparatus and its ecofriendly dyeing production," Journal of CO2 Utilization, vol. 16, pp. 272-281, 2016.

[4] J.-J. Long, C.-L. Cui, Y.-Q. Zhang, and G.-H. Yuan, "Clean fixation of dye on cotton in supercritical carbon dioxide with a heterogeneous and phase transfer catalytic reaction," Dyes and Pigments, vol. 115, pp. 88-95, 2015.

[5] T. A. Elmaaty, E. A. El-Aziz, J. Ma, F. El-Taweel, and S. Okubayashi, "Eco-friendly disperse dyeing and functional finishing of nylon 6 using supercritical carbon dioxide," Fibers, vol. 3, no. 4, pp. 309-322, 2015.

[6] J.-J. Long, Y.-Q. Ma, and J.-P. Zhao, "Investigations on the level dyeing of fabrics in supercritical carbon dioxide," The Journal of Supercritical Fluids, vol. 57, no. 1, pp. 80-86, 2011.

[7] A. R. Horrocks and S. C. Anand, Handbook of Technical Textiles, vol. 1, ch. 9, Elsevier, 2016.

[8] V. K. Gupta, "Application of low-cost adsorbents for dye removal - A review," Journal of Environmental Management, vol. 90, no. 8, pp. 2313-2342, 2009.

[9] G. Phillips and P. Williams, Handbook of Hydrocolloids, vol. 1, ch. 12, Woodhead Publishing, 2009.

[10] A. M. Stephen, G. O. Phillips, and P. A. Williams, Food Polysaccharides and Their Applications, CRC Press, second edition, 2006.

[11] M. Khotimchenko, K. Makarova, E. Khozhaenko, and V. Kovalev, "Lead binding capacity of calcium pectates with different molecular weight," International Journal of Biological Macromolecules, vol. 97, pp. 526-535, 2017.

[12] A. A. Kadam, J. Jang, and D. S. Lee, "Facile synthesis of pectin-stabilized magnetic graphene oxide Prussian blue 
nanocomposites for selective cesium removal from aqueous solution," Bioresource Technology, vol. 216, pp. 391-398, 2016.

[13] J.-L. Gong, X.-Y. Wang, G.-M. Zeng et al., “Copper (II) removal by pectin-iron oxide magnetic nanocomposite adsorbent," Chemical Engineering Journal, vol. 185-186, pp. 100-107, 2012.

[14] M. Y. Khotimchenko, E. A. Kolenchenko, Y. S. Khotimchenko, E. V. Khozhaenko, and V. V. Kovalev, "Cerium binding activity of different pectin compounds in aqueous solutions," Colloids and Surfaces. B, Biointerfaces, vol. 77, no. 1, pp. 104-110, 2010.

[15] Y. Mata, M. Blázquez, A. Ballester, F. González, and J. A. Muñoz, "Sugar-beet pulp pectin gels as biosorbent for heavy metals: Preparation and determination of biosorption and desorption characteristics," Chemical Engineering Journal, vol. 150, no. 2-3, pp. 289-301, 2009.

[16] M. Khotimchenko, E. Kolenchenko, and Y. Khotimchenko, "Zinc-binding activity of different pectin compounds in aqueous solutions," Journal of Colloid and Interface Science, vol. 323, no. 2, pp. 216-222, 2008.

[17] M. F. Abdullah, A. Azfaralariff, and A. M. Lazim, "Methylene blue removal by using pectin-based hydrogels extracted from dragon fruit peel waste using gamma and microwave radiation polymerization techniques," Journal of Biomaterials Science, Polymer Edition, vol. 29, no. 14, pp. 1745-1763, 2018.

[18] N. R. Singha, M. Karmakar, M. Mahapatra et al., "Systematic synthesis of pectin-g-(sodium acrylate-co-N-isopropylacrylamide) interpenetrating polymer network for superadsorption of dyes/M(ii): determination of physicochemical changes in loaded hydrogels," Polymer Chemistry, vol. 8, no. 20, pp. 3211-3237, 2017.

[19] D. Li, J. Wang, Z. Guo, J. Li, and J. Shuai, "Pectin gels crosslinked by $\mathrm{Ca}^{2}{ }^{+}$: An efficient material for methylene blue removal," Journal of Molecular Liquids, vol. 238, pp. 36-42, 2017.

[20] R. Rakhshaee and Y. Noorani, "Comparing three methods of simultaneous synthesis and stabilization of $\mathrm{Fe} 3 \mathrm{O} 4$ nanoparticles: changing physicochemical properties of products to improve kinetic and thermodynamic of dye adsorption," Journal of Magnetism and Magnetic Materials, vol. 422, pp. 128-140, 2017.

[21] V. K. Gupta, D. Pathania, and P. Singh, "Pectin-cerium (IV) tungstate nanocomposite and its adsorptional activity for removal of methylene blue dye," International journal of Environmental Science and Technology, vol. 11, no. 7, pp. 20152024, 2014.

[22] R. Rakhshaee and M. Panahandeh, "Stabilization of a magnetic nano-adsorbent by extracted pectin to remove methylene blue from aqueous solution: A comparative studying between two kinds of cross-likened pectin," Journal of Hazardous Materials, vol. 189, no. 1-2, pp. 158-166, 2011.

[23] O. A. Attallah, M. A. Al-Ghobashy, M. Nebsen, and M. Y. Salem, "Removal of cationic and anionic dyes from aqueous solution with magnetite/pectin and magnetite/silica/pectin hybrid nanocomposites: kinetic, isotherm and mechanism analysis," RSC Advances, vol. 6, no. 14, pp. 11461-11480, 2016.

[24] A. R. Nesic, S. J. Velickovic, and D. G. Antonovic, "Novel composite films based on amidated pectin for cationic dye adsorption," Colloids and Surfaces B: Biointerfaces, vol. 116, pp. 620626, 2014.
[25] R. Rakhshaee, M. Giahi, and A. Pourahmad, "Removal of methyl orange from aqueous solution by _Azolla filicoloides_: Synthesis of $\mathrm{Fe}_{3} \mathrm{O}_{4}$ nano-particles and its surface modification by the extracted pectin of Azolla," Chinese Chemical Letters, vol. 22, no. 4, pp. 501-504, 2011.

[26] M. Li and G. Buschle-Diller, "Pectin-blended anionic polysaccharide films for cationic contaminant sorption from water," International Journal of Biological Macromolecules, vol. 101, pp. 481-489, 2017.

[27] I. Braccini and S. Pérez, "Molecular basis of Ca2+-induced gelation in alginates and pectins: the egg-box model revisited," Biomacromolecules, vol. 2, no. 4, pp. 1089-1096, 2001.

[28] I. Langmuir, "The constitution and fundamental properties of solids and liquids. Part I. Solids," Journal of the American Chemical Society, vol. 38, no. 11, pp. 2221-2295, 1916.

[29] H. Freundlich, "Über die Adsorption in Lösungen," Zeitschrift für Physikalische Chemie, vol. 57, pp. 385-470, 1907.

[30] B. H. Zimm and J. K. Bragg, "Theory of the phase transition between helix and random coil in polypeptide chains," The Journal of Chemical Physics, vol. 31, no. 2, pp. 526-535, 1959.

[31] R Core Team, R: A Language and Environment for Statistical Computing, R Foundation for Statistical Computing, Vienna, Austria, 2020.

[32] J. A. Lozano-Álvarez, V. F. Maraňon Ruiz, J. Jáuregui-Rincón, I. Medina- Ramírez, R. Salinas-Gutíerrez, and C. FraustoReyes, "Remoción de colorantes azo con alginato: Relación entre estructura de colorante y eficiencia de remoción," Revista Internacional de Contaminacion Ambiental, vol. 35, no. 1, pp. 223-236, 2019.

[33] I. Braccini, R. P. Grasso, and S. Pérez, "Conformational and configurational features of acidic polysaccharides and their interactions with calcium ions: a molecular modeling investigation," Carbohydrate Research, vol. 317, no. 1-4, pp. 119130, 1999.

[34] J. A. Lozano-Alvarez, V.-F. Maraňon Ruiz, J. Jáuregui-Rincón, I. Medina-Ramírez, C. Frausto-Reyes, and R. Salinas-Gutíerrez, "Removal of direct dyes with alginic acid," Journal of the Mexican Chemical Society, vol. 59, no. 3, pp. 215-228, 2015.

[35] J. A. Lozano-Alvarez, J. Jauregui-Rincon, G. Mendoza-Díaz, R. Rodríguez-Vázquez, and C. Frausto-Reyes, "Study of sorption equilibrium of biopolymers alginic acid and xanthan with C.I. disperse yellow 54," Journal of the Mexican Chemical Society, vol. 53, no. 2, pp. 59-70, 2019.

[36] J. Oakes and S. Dixon, "Physical interactions of dyes in solution - influence of dye structure on aggregation and binding to surfactants/polymers," Review of Progress in Coloration and Related Topics, vol. 34, no. 1, pp. 110-128, 2004.

[37] M. Clark, "Principles, Processes and Types of Dyes of Woodhead Publishing Series in Textiles," in Handbook of Textile and Industrial Dyeing, Woodhead Publishing, 2011.

[38] R. Sabnis, Handbook of Acid-Base Indicators, CRC Press, 2007.

[39] D. Ibarra-Rodríguez, J. Lizardi-Mendoza, E. A. Lopez-Maldonado, and M. T. Oropeza-Guzman, "Capacity of 'nopal' pectin as a dual coagulant-flocculant agent for heavy metals removal," Chemical Engineering Journal, vol. 323, pp. 19-28, 2017.

[40] Y. Ho, I. Norli, A. F. Alkarkhi, and N. Morad, "Characterization of biopolymeric flocculant (pectin) and organic synthetic flocculant (PAM): a comparative study on treatment and optimization in kaolin suspension," Bioresource Technology, vol. 101, pp. 1166-1174, 2010. 
[41] A. Jagusiak, B. Piekarska, T. Pańczyk et al., "Dispersion of single-wall carbon nanotubes with supramolecular Congo red - properties of the complexes and mechanism of the interaction," Beilstein Journal of Nanotechnology, vol. 8, pp. 636648, 2017.

[42] H. Wang, L. Wan, D. Chen, X. Guo, F. Liu, and S. Pan, "Unexpected gelation behavior of citrus pectin induced by monovalent cations under alkaline conditions," Carbohydrate Polymers, vol. 212, pp. 51-58, 2019.

[43] M. Muttakin, S. Mitra, K. Thu, K. Ito, and B. B. Saha, “Theoretical framework to evaluate minimum desorption temperature for IUPAC classified adsorption isotherms," International Journal of Heat and Mass Transfer, vol. 122, pp. 795-805, 2018.

[44] G. Limousin, J.-P. Gaudet, L. Charlet, S. Szenknect, V. Barthes, and M. Krimissa, "Sorption isotherms: a review on physical bases, modeling and measurement," Applied Geochemistry, vol. 22, no. 2, pp. 249-275, 2007.

[45] C. Hinz, "Description of sorption data with isotherm equations," Geoderma, vol. 99, no. 3, pp. 225-243, 2001.

[46] M. A. Al-Ghouti and D. A. Da'ana, "Guidelines for the use and interpretation of adsorption isotherm models: a review," Journal of Hazardous Materials, vol. 393, article 122383, 2020.

[47] J. A. Lozano-Alvarez, J. Jauregui-Rincon, I. Medina-Ramirez, C. Frausto- Reyes, and R. Salinas-Gutierrez, "Removal of azo dyes with xanthan," Journal of the Mexican Chemical Society, vol. 63, no. 4, pp. 176-198, 2019.

[48] J. Jauregui-Rincon, J. A. Lozano-Alvarez, and I. MedinaRamirez, "Zimmbragg model applied to sorption of dyes by biopolymers: alginic acid and xanthan," in Biotechnology of Biopolymers, M. Elnashar, Ed., pp. 165-190, InTech, 2011.

[49] K. Hayakawa and J. C. T. Kwak, "Surfactant-polyelectrolyte interactions. 1. Binding of dodecyltrimethylammonium ions by sodium dextransulfate and sodium poly(styrenesulfonate) in aqueous solution in the presence of sodium chloride," The Journal of Physical Chemistry, vol. 86, no. 19, pp. 3866-3870, 1982.

[50] K. Hayakawa and J. C. T. Kwak, "Study of surfactantpolyelectrolyte interactions. 2. Effect of multivalent counterions on the binding of dodecyltrimethylammonium ions by sodium dextran sulfate and sodium poly(styrene sulfonate) in aqueous solution," The Journal of Physical Chemistry, vol. 87, no. 3, pp. 506-509, 1983.

[51] A. Malovikova, K. Hayakawa, and J. C. T. Kwak, "Surfactant polyelectrolyte interactions. 4. Surfactant chain length dependence of the binding of alkylpyridinium cations to dextran sulfate," The Journal of Physical Chemistry, vol. 88, no. 10, pp. 1930-1933, 1984.

[52] K. Hayakawa, J. P. Santerre, and J. C. T. Kwak, "Study of surfactant polyelectrolyte interactions. Binding of dodecyl- and tetradecyltrimethylammonium bromide by some carboxylic polyelectrolytes," Macromolecules, vol. 16, no. 10, pp. 1642$1645,1983$.

[53] E. D. Goddard and J. V. Gruber, Principles of Polymer Science and Technology in Cosmetics and Personal Care, CRC Press, 1999.

[54] M. Kobayashi, A. Sasagawa, T. Hoshi et al., "High-order aggregation of crystal violet in the chromonic lyotropic mesophases of 7,7'-disodiumchromoglycate," Molecular Crystals and Liquid Crystals Science and Technology. Section A. Molecular Crystals and Liquid Crystals, vol. 225, no. 1, pp. 293-301, 1993.
[55] L.-L. Yu, L.-N. Jiang, S.-Y. Wang, M.-M. Sun, D.-Q. Li, and G.$\mathrm{M} . \mathrm{Du}$, "Pectin microgel particles as high adsorption rate material for methylene blue: performance, equilibrium, kinetic, mechanism and regeneration studies," International Journal of Biological Macromolecules, vol. 112, pp. 383-389, 2018.

[56] E. F. Lessa, M. S. Gularte, E. S. Garcia, and A. R. Fajardo, "Orange waste: a valuable carbohydrate source for the development of beads with enhanced adsorption properties for cationic dyes," Carbohydrate Polymers, vol. 157, pp. 660-668, 2017.

[57] A. K. Kodoth and V. Badalamoole, "Silver nanoparticleembedded pectin-based hydrogel for adsorptive removal of dyes and metal ions," Polymer Bulletin, vol. 77, pp. 541-564, 2020.

[58] A. Mirza and R. Ahmad, "An efficient sequestration of toxic crystal violet dye from aqueous solution by alginate/pectin nanocomposite: a novel and ecofriendly adsorbent," Groundwater for Sustainable Development, vol. 11, article 100373, 2020.

[59] J. Oakes and P. Gratton, "Kinetic investigations of azo dye oxidation in aqueous media," Journal of the Chemical Society, Perkin Transactions, vol. 2, no. 9, pp. 1857-1864, 1998.

[60] A. Hassanzadeh, A. Zeini-Isfahani, M. Habibi, M. Poor Heravi, and M. Abdollahi-Alibeik, "1H, 13C, N-H, H-H, C-H COSY, H-H NOESY NMR and UV-vis studies of Solophenyl red 3BL dye azo-hydrazone tautomerism in various solvents," Spectrochimica Acta Part A: Molecular and Biomolecular Spectroscopy, vol. 63, no. 2, pp. 247-254, 2006.

[61] L. C. Abbott, S. N. Batchelor, L. Jansen, J. Oakes, J. R. Lindsay Smith, and J. N. Moore, "Spectroscopic studies of direct blue 1 in solution and on cellulose surfaces: effects of environment on a bis-azo dye," New Journal of Chemistry, vol. 28, no. 7, pp. 815-821, 2004.

[62] M. Dakiky, K. Kanan, and M. Khamis, "Aggregation of o, ódihydroxyazo dyes II. Interaction of 2-hydroxy-4-nitrophenylazoresorcinol in DMSO and DMF," Dyes and Pigments, vol. 41, no. 3, pp. 199-209, 1999.

[63] O. N. Makshakova, T. A. Gorshkova, P. V. Mikshina, Y. F. Zuev, and S. Perez, "Metrics of rhamnogalacturonan I with $\beta$ $-(1 \longrightarrow 4)$ s-linked galactan side chains and structural basis for its self-aggregation," Carbohydrate Polymers, vol. 158, pp. 93-101, 2017.

[64] S. Perez, M. A. Rodriguez-Carvajal, and T. Doco, "A complex plant cell wall polysaccharide: rhamnogalacturonan II. A structure in quest of a function," Biochimie, vol. 85 , no. 1-2, pp. 109-121, 2003.

[65] L. Cao, W. Lu, A. Mata, K. Nishinari, and Y. Fang, "Egg-box model based gelation of alginate and pectin: a review," Carbohydrate Polymers, vol. 242, article 116389, 2020.

[66] Q. Zhou, C. Xie, W. Gong, N. Xu, and W. Zhou, "Comments on the method of using maximum absorption wavelength to calculate Congo red solution concentration," Journal of Hazardous Materials, vol. 198, pp. 381-382, 2011.

[67] L. Wang, J. Li, Z. Wang, L. Zhao, and Q. Jiang, "Low-temperature hydrothermal synthesis of $\alpha-\mathrm{Fe} / \mathrm{Fe}_{3} \mathrm{O}_{4}$ nanocomposite for fast Congo red removal," Dalton Transactions, vol. 42, no. 7, pp. 2572-2579, 2013.

[68] Z. L. Yaneva and N. V. Georgieva, "Insights into Congo red adsorption on agro-industrial materials - spectral, equilibrium, kinetic, thermodynamic, dynamic and desorption studies. A 
review," International Review of Chemical Engineering, vol. 4, no. 2, pp. 127-146, 2012.

[69] J. Barkauskas, I. Stankeviciene, J. Daksevic, and A. Padarauskas, "Interaction between graphite oxide and Congo red in aqueous media," Carbon, vol. 49, no. 15, pp. 5373-5381, 2011.

[70] M. Movahedi, A. R. Mahjoub, and S. Janitabar-Darzi, "Photodegradation of Congo red in aqueous solution on $\mathrm{ZnO}$ as an alternative catalyst to TiO2," Journal of the Iranian Chemical Society, vol. 6, no. 3, pp. 570-577, 2009.

[71] E. Pigorsch, A. Elhaddaoui, and S. Turrell, "Spectroscopic study of $\mathrm{pH}$ and solvent effects on the structure of Congo red and its binding mechanism to amyloid-like proteins," Spectrochimica Acta Part A: Molecular Spectroscopy, vol. 50, no. 12, pp. 2145-2152, 1994.

[72] P. Spolnik, B. Stopa, B. Piekarska et al., "The use of rigid, fibrillar Congo red nanostructures for scaffolding protein assemblies and inducing the formation of amyloid-like arrangement of molecules," Chemical Biology \& Drug Design, vol. 70, no. 6, pp. 491-501, 2007.

[73] M.-C. Ralet, V. Dronnet, H. C. Buchholt, and J.-F. Thibault, "Enzymatically and chemically de-esterified lime pectins: characterisation, polyelectrolyte behaviour and calcium binding properties," Carbohydrate Research, vol. 336, no. 2, pp. 117-125, 2001.

[74] K. M. Tawarah and H. M. Abu-Shamleh, "A spectrophotometric study of the tautomeric and acid-base equilibria of methyl orange and methyl yellow in aqueous acidic solutions," Dyes and Pigments, vol. 16, no. 3, pp. 241-251, 1991.

[75] J. Del Nero, R. E. de Araujo, A. S. L. Gomes, and C. P. de Melo, "Theoretical and experimental investigation of the second hyperpolarizabilities of methyl orange," The Journal of Chemical Physics, vol. 122, no. 10, article 104506, 2005.

[76] M. De Vylder and D. De Keukeleire, "Aggregation of methyl orange in aqueous acidic solution," Bulletin des Societes Chimiques Belges, vol. 87, no. 1, pp. 9-13, 1978.

[77] C. Nancoz, G. Licari, J. S. Beckwith et al., "Influence of the hydrogen-bond interactions on the excited-state dynamics of a push-pull azobenzene dye: the case of methyl orange," Physical Chemistry Chemical Physics, vol. 20, no. 10, pp. 72547264, 2018.

[78] R. L. Reeves, R. S. Kaiser, M. S. Maggio, E. A. Sylvestre, and W. H. Lawton, "Analysis of the visual spectrum of methyl orange in solvents and in hydrophobic binding sites," Canadian Journal of Chemistry, vol. 51, no. 4, pp. 628-635, 1973.

[79] K. M. Tawarah and H. M. Abu-Shamleh, "A spectrophotometric study of the acid-base equilibria of o-methyl red in aqueous solutions," Dyes and Pigments, vol. 17, no. 3, pp. 203-215, 1991.

[80] M. Garcia-Heras, K. Kromka, J. Faber, P. Karaszkiewicz, and M. A. Villegas, "Evaluation of air acidity through optical sensors," Environmental Science \& Technology, vol. 39, no. 10, pp. 3743-3747, 2005.

[81] K. M. Tawarah, "The distribution diagram and the visible spectra of o-methyl red species in aqueous solutions," Dyes and Pigments, vol. 18, no. 3, pp. 237-249, 1992.

[82] S.-K. Park, C. Lee, K.-C. Min, and N.-S. Lee, "Fourier transform Raman studies of methyl red adsorbed on -alumina and silica-alumina," Bulletin of the Korean Chemical Society, vol. 25, no. 12, pp. 1817-1821, 2004.
[83] K. M. Tawarah and A. A. Wazwaz, "The electrical conductivities of the sodium salts of methyl orange, o-methyl red and $\mathrm{p}$ methyl red in aqueous solutions," Dyes and Pigments, vol. 21, no. 2, pp. 97-103, 1993.

[84] R. S. Blackburn, "Natural polysaccharides and their interactions with dye molecules: applications in effluent treatment," Environmental Science \& Technology, vol. 38, no. 18, pp. 4905-4909, 2004.

[85] B. Schrader, Ed., Infrared and Raman Spectroscopy: Methods and Applications, VCH Verlagsgesellschaft mbH, 1995.

[86] R. M. Silverstein, F. X. Webster, D. J. Kiemle, and D. L. Bryce, Spectrometric Identification of Organic Compounds, Wiley, 8th edition, 2014.

[87] J.-A. He, S. Bian, L. Li, J. Kumar, S. K. Tripathy, and L. A. Samuel-son, "Photochemical behavior and formation of surface relief grating on self-assembled polyion/dye composite film," The Journal of Physical Chemistry B, vol. 104, no. 45, pp. 10513-10521, 2000.

[88] D. R. Armstrong, J. Clarkson, and W. E. Smith, "Vibrational analysis of trans-azobenzene," The Journal of Physical Chemistry, vol. 99, no. 51, pp. 17825-17831, 1995.

[89] N. Biswas and S. Umapathy, "Structures, vibrational frequencies, and normal modes of substituted azo dyes: infrared, Raman, and density functional calculations," The Journal of Physical Chemistry A, vol. 104, no. 12, pp. 2734-2745, 2000.

[90] C. E. Bonancêa, G. M. do Nascimento, M. L. De Souza, M. L. Temperini, and P. Corio, "Substrate development for surface-enhanced Raman study of photocatalytic degradation processes: Congo red over silver modified titanium dioxide films," Applied Catalysis B: Environmental, vol. 69, no. 1-2, pp. 34-42, 2006.

[91] H. Lee, K. Machida, A. Kuwae, and Y. Saito, "Resonance raman spectra and structure of _p_-aminoazobenzene dyes in diprotonated form," Journal of Molecular Structure, vol. 68 , pp. 51-57, 1980. 